Supporting Information

for

\title{
Synthesis and Pharmacological Evaluation of Huprine-Tacrine Heterodimers, Subnanomolar Dual Binding Site Acetylcholinesterase Inhibitors
}

\author{
Pelayo Camps, ${ }^{\mathrm{a}, *}$ Xavier Formosa, ${ }^{\mathrm{a}}$ Diego Muñoz-Torrero, ${ }^{\mathrm{a},{ }^{*}}$ Julien Petrignet $^{\mathrm{a}, \mathrm{b}}$, \\ Albert Badia, ${ }^{\mathrm{c}}$ and Maria Victòria $\operatorname{Clos}^{\mathrm{c}}$
}

\footnotetext{
a Laboratori de Química Farmacèutica (Unitat Associada al CSIC), Facultat de Farmàcia, Universitat de Barcelona, Av. Diagonal 643, E-08028, Barcelona, Spain

${ }^{\mathrm{b}}$ Current address: Laboratoire de Synthèse Organométallique et Réactivité, Faculté des Sciences et Techniques, Université Henri Poincaré, Bd. des Aiguillettes, 54506-Vandoeuvre les Nancy, France

${ }^{\mathrm{c}}$ Departament de Farmacologia i Terapèutica, Facultat de Medicina, Universitat Autònoma de Barcelona, E-08193-Bellaterra, Barcelona, Spain
}

Supporting information available: Experimental part (chemistry and pharmacology) (Total 24 pages). 
Chemistry. General Methods. Melting points were determined in open capillary tubes with a MFB 595010M Gallenkamp melting point apparatus. $300 \mathrm{MHz}{ }^{1} \mathrm{H} / 75.4{ }^{13} \mathrm{C}$ NMR spectra, $400 \mathrm{MHz}{ }^{1} \mathrm{H} / 100.6{ }^{13} \mathrm{C}$ NMR spectra, and $500 \mathrm{MHz}{ }^{1} \mathrm{H}$ NMR spectra were recorded on Varian Gemini 300, Varian Mercury 400, and Varian Inova 500 or Brucker DMX500 spectrometers, respectively. The chemical shifts are reported in ppm ( $\delta$ scale) relative to internal TMS, and coupling constants are reported in Hertz (Hz). Assignments given for the NMR spectra of the new compounds have been carried out by comparison with the NMR data of $\mathbf{1}, \mathbf{6}, \mathbf{8}, 13$ and $\mathbf{1 5 a} \cdot \mathrm{HCl}$, as model compounds, which in turn, were assigned on the basis of DEPT, COSY ${ }^{1} \mathrm{H} /{ }^{1} \mathrm{H}$ (standard procedures), and COSY ${ }^{1} \mathrm{H} /{ }^{13} \mathrm{C}$ (gHMQC, gHSQC or gHMBC sequences) experiments. The syn (anti) notation of the protons at position 13 of compounds $\mathbf{6}, \mathbf{8}, \mathbf{1 2}, \mathbf{1 7} \mathbf{a}-\mathbf{d}$, and $\mathbf{1 8 a}-\mathbf{d}$ means that the corresponding proton at position 13 is on the same (different) side of the quinoline moiety with respect to the cyclohexene ring. HRMS were performed on a Micromass Autospec spectrometer. IR spectra were run on a FT/IR Perkin-Elmer model 1600 spectrophotometer. Absorption values are expressed as wave-numbers $\left(\mathrm{cm}^{-1}\right)$; only significant absorption bands are given. Column chromatography was performed on silica gel 60 AC.C (35-70 mesh, SDS, ref 2000027 or 70-200 mesh, SDS, ref 2100027). Thin-layer chromatography (TLC) was performed with aluminum-backed sheets with silica gel $60 \mathrm{~F}_{254}$ (Merck, ref 1.05554), and spots were visualized with UV light and $1 \%$ aqueous solution of $\mathrm{KMnO}_{4}$. Analytical grade solvents were used for crystallization, while pure for synthesis solvents were used in the reactions, extractions and column chromatography. For characterization 
purposes, the new compounds $16 \mathbf{a}-\mathbf{c}$ and $\mathbf{1 5 d}$ and $\mathbf{1 6 d}$ were transformed into the corresponding hydrobromides or dihydrochlorides, respectively, and recrystallized, and the new huprine-tacrine heterodimers were also characterized as dihydrochlorides or trihydrochlorides (in the case of $\mathbf{1 7 d}$ and $\mathbf{1 8 d}$ ). Worthy of note, as previously reported for some tacrine-related dimeric compounds, ${ }^{19}$ new huprine-tacrine heterodimers have the ability to retain molecules of water and of solvents of crystallization, which can not be removed after drying the analytical samples at $80{ }^{\circ} \mathrm{C} / 1$ Torr for 2 days. Thus, the elemental analyses of these compounds showed the presence of variable amounts of water and in some cases of AcOEt, used in the crystallization. NMR spectra of all of the new compounds were performed at the Serveis Científico-Tècnics of the University of Barcelona, while high resolution mass spectra and elemental analyses were carried out at the Mass Spectrometry Laboratory of the University of Santiago de Compostela (Spain) and at the Mycroanalysis Service of the IIQAB (CSIC, Barcelona, Spain), respectively.

\section{9-Ethyl-6,7,10,11-tetrahydro-7,11-methano-5H-cycloocta[b]quinolin-12-one $\quad[( \pm)-6]$.}

Enone $( \pm)-5(1.00 \mathrm{~g}, 6.10 \mathrm{mmol})$ was added to a suspension of anthranilic acid (1.00 g, 7.29 mmol, 1.2 equiv.) in toluene ( $5 \mathrm{~mL})$, and the reaction mixture was heated under reflux for 2 $\mathrm{h}$ with azeotropic removal of water. Filtration in vacuo of the cold $\left(0{ }^{\circ} \mathrm{C}\right)$ suspension, followed by successive washings of the solid with toluene $(6 \mathrm{~mL})$ and a mixture EtOH / $\mathrm{Et}_{2} \mathrm{O}$ 1:1 (6 mL) gave a regioisomeric mixture of quinolones $( \pm)-6$ and $( \pm)-7$ in an approximate ratio of $90: 10\left({ }^{1} \mathrm{H}\right.$ NMR) $(1.10 \mathrm{~g}, 68 \%$ yield). Sublimation of this crude product at $230{ }^{\circ} \mathrm{C} / 0.8$ Torr afforded a mixture $( \pm)-6$ / $( \pm)-7$ in a ratio of 93:7 as a yellowish 
solid: mp 295-296 ${ }^{\circ} \mathrm{C} ; R_{f} 0.37\left(\mathrm{SiO}_{2}\right.$, AcOEt); IR (KBr) $v 3413$ (NH st), 1634 (C=O st) $\mathrm{cm}^{-1}$; NMR data of the main regioisomer, $( \pm)$-6, from the spectra of the mixture $( \pm)-6$ / $( \pm)$ 7 in the ratio of 93:7: ${ }^{1} \mathrm{H}$ NMR (500 MHz, $\left.\mathrm{CD}_{3} \mathrm{OD}\right) \delta 0.87\left(\mathrm{t}, J=7.5 \mathrm{~Hz}, 3 \mathrm{H}, \mathrm{CH}_{2} \mathrm{CH}_{3}\right.$ ), 1.79 (dm, $J=12.0 \mathrm{~Hz}, 1 \mathrm{H}, 13-\mathrm{H}_{\text {syn }}$ ), superimposed in part 1.81-1.89 (complex signal, $2 \mathrm{H}$, $\mathrm{CH}_{2} \mathrm{CH}_{3}$ ), 1.94 (dddd, $J=12.0 \mathrm{~Hz}, J^{\prime}=4.0 \mathrm{~Hz}, J^{\prime \prime}=J^{\prime \prime \prime}=2.0 \mathrm{~Hz}, 1 \mathrm{H}, 13-\mathrm{H}_{\text {anti }}$ ), 2.07 (broad d, $\left.J=17.5 \mathrm{~Hz}, 1 \mathrm{H}, 10-\mathrm{H}_{\text {endo }}\right), 2.40\left(\mathrm{ddm}, J=17.5 \mathrm{~Hz}, J^{\prime}=5.5 \mathrm{~Hz}, 1 \mathrm{H}, 10-\mathrm{H}_{\text {exo }}\right.$ ), 2.64 (ddd, $\left.J=17.0 \mathrm{~Hz}, J^{\prime}=J^{\prime \prime}=2.0 \mathrm{~Hz}, 1 \mathrm{H}, 6-\mathrm{H}_{\text {endo }}\right), 2.70(\mathrm{~m}, 1 \mathrm{H}, 7-\mathrm{H}), 3.04\left(\mathrm{dd}, J=17.0 \mathrm{~Hz}, J^{\prime}=6.0 \mathrm{~Hz}\right.$, 1H, 6- $\mathrm{H}_{\text {exo }}$ ), 3.51 (m, 1H, 11-H), 5.50 (dm, $\left.J=5.5 \mathrm{~Hz}, 1 \mathrm{H}, 8-\mathrm{H}\right), 7.31$ (ddd, $J=8.0 \mathrm{~Hz}, J^{\prime}=7.0$ Hz, $J^{\prime \prime}=1.5 \mathrm{~Hz}, 1 \mathrm{H}, 2-\mathrm{H}$ ), 7.46 (broad d, $J=8.5 \mathrm{~Hz}, 1 \mathrm{H}, 4-\mathrm{H}$ ), 7.60 (ddd, $J=8.5 \mathrm{~Hz}, J^{\prime}=7.0$ $\left.\mathrm{Hz}, J^{\prime \prime}=1.5 \mathrm{~Hz}, 1 \mathrm{H}, 3-\mathrm{H}\right), 8.23$ (dd, $\left.J=8.5 \mathrm{~Hz}, J^{\prime}=1.5 \mathrm{~Hz}, 1 \mathrm{H}, 1-\mathrm{H}\right) ;{ }^{13} \mathrm{C}$ NMR $(100.6 \mathrm{MHz}$, $\left.\mathrm{CD}_{3} \mathrm{OD}\right) \delta 12.7\left(\mathrm{CH}_{3}, \mathrm{CH}_{2} \mathrm{CH}_{3}\right), 26.9(\mathrm{CH}, \mathrm{C} 11), 28.9(\mathrm{CH}, \mathrm{C} 7), 30.1\left(\mathrm{CH}_{2}, \mathrm{C} 13\right), 31.1$ ( $\left.\mathrm{CH}_{2}, \mathrm{CH}_{2} \mathrm{CH}_{3}\right), 35.4\left(\mathrm{CH}_{2}, \mathrm{C} 10\right), 35.7\left(\mathrm{CH}_{2}, \mathrm{C} 6\right), 118.5(\mathrm{CH}, \mathrm{C} 4), 122.0(\mathrm{C})$ and 125.1 (C) (C11a and C12a), 123.3 (CH, C8), 124.2 (CH, C2), 126.2 (CH, C1), 132.7 (CH, C3), 140.8 (C), 141.0 (C), and 149.7 (C) (C4a, C5a, and C9), 178.4 (C, C12). Anal. $\left(\mathrm{C}_{18} \mathrm{H}_{19} \mathrm{NO} \cdot 0.4 \mathrm{H}_{2} \mathrm{O}\right) \mathrm{C}, \mathrm{H}, \mathrm{N}$.

12-Chloro-9-ethyl-6,7,10,11-tetrahydro-7,11-methanocycloocta[b]quinoline $[( \pm)-8]$. A 9:1 mixture of quinolones $( \pm)-6$ / $( \pm)-7(1.50 \mathrm{~g}, 5.09 \mathrm{mmol})$ was added in portions to $\mathrm{POCl}_{3}$ (5.18 mL, $8.52 \mathrm{~g}, 55.6 \mathrm{mmol}, 10.9$ equiv.) (CAUTION: exothermic reaction), and the reaction mixture was heated under reflux for $2 \mathrm{~h}$. The resulting suspension was cooled to room temperature and was added dropwise to a solution of $\mathrm{KOH}(28 \mathrm{~g})$ in a mixture water / 
ice $(50 \mathrm{~mL})$ under vigorous stirring. $\mathrm{CH}_{2} \mathrm{Cl}_{2}(75 \mathrm{~mL})$ was added to the mixture, the organic phase was separated, and the aqueous one was extracted with $\mathrm{CH}_{2} \mathrm{Cl}_{2}(3 \times 50 \mathrm{~mL})$. The combined organic extracts were dried with anhydrous $\mathrm{Na}_{2} \mathrm{SO}_{4}$, and evaporated at reduced pressure to give a yellowish solid residue $(1.44 \mathrm{~g})$, which was submitted to column chromatography (70-200 mesh silica gel, 144 g, hexane / AcOEt mixtures). On elution with hexane / AcOEt 95:5, chloroquinoline ( \pm )-8 (892 mg, 62\% yield) was isolated as a light yellow solid. On elution with hexane / AcOEt 90:10, a mixture of $( \pm)-\mathbf{8} /( \pm)-\mathbf{9}$ in the approximate ratio of 10:1 ( ${ }^{1} \mathrm{H}$ NMR) $(170 \mathrm{mg})$, and mixtures of $( \pm)-\mathbf{8} /( \pm)-\mathbf{9} /( \pm)-\mathbf{1 0}$ in the approximate ratios of 82:9:8 (142 $\mathrm{mg})$ and 8:48:43 (120 mg) [81\% total yield of $( \pm)-8$ ] were successively isolated.

( \pm )-8: mp $86-87^{\circ} \mathrm{C}$ (sublimed at $140{ }^{\circ} \mathrm{C} / 0.5$ Torr); $R_{f} 0.55\left(\mathrm{SiO}_{2}\right.$, hexane / AcOEt 1:1); IR (KBr) $v$ 1616, 1580, and 1554 (ar-C-C and ar-C-N st) cm ${ }^{-1} ;{ }^{1} \mathrm{H}$ NMR (500 MHz, $\left.\mathrm{CDCl}_{3}\right) \delta$ 0.84 (t, $\left.J=7.5 \mathrm{~Hz}, 3 \mathrm{H}, \mathrm{CH}_{2} \mathrm{CH}_{3}\right), 1.76$ (dq, $\left.J=15.0 \mathrm{~Hz}, J^{\prime}=7.5 \mathrm{~Hz}, 1 \mathrm{H}\right)$ and 1.80 (dq, $J=15.0$ $\left.\mathrm{Hz}, J^{\prime}=7.5 \mathrm{~Hz}, 1 \mathrm{H}\right)\left(\mathrm{CH}_{2} \mathrm{CH}_{3}\right), 1.96\left(\mathrm{dm}, J=12.5 \mathrm{~Hz}, 1 \mathrm{H}, 13-\mathrm{H}_{\text {syn }}\right)$, superimposed in part 2.07 (dddd, $J=12.5 \mathrm{~Hz}, J^{\prime}=4.5 \mathrm{~Hz}, J^{\prime \prime}=J^{\prime \prime \prime}=2.0 \mathrm{~Hz}, 1 \mathrm{H}, 13-\mathrm{H}_{a n t i}$ ), superimposed in part 2.10

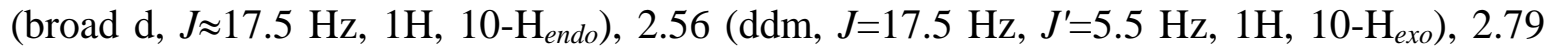
(m, 1H, 7-H), 3.11 (dm, J=18.0 Hz, 1H, 6-H endo), 3.20 (dd, J=18.0 Hz, J'=6.0 Hz, 1H, 6$\mathrm{H}_{\text {exo }}$ ), 3.77 (m, 1H, 11-H), 5.51 (dm, $\left.J=5.5 \mathrm{~Hz}, 1 \mathrm{H}, 8-\mathrm{H}\right), 7.52$ (ddd, $J=8.5 \mathrm{~Hz}, J^{\prime}=7.0 \mathrm{~Hz}$, $\left.J^{\prime \prime}=1.5 \mathrm{~Hz}, 1 \mathrm{H}, 2-\mathrm{H}\right), 7.65$ (ddd, $\left.J=8.5 \mathrm{~Hz}, J^{\prime}=7.0 \mathrm{~Hz}, J^{\prime \prime}=1.5 \mathrm{~Hz}, 1 \mathrm{H}, 3-\mathrm{H}\right), 7.97$ (broad d, $J=8.5 \mathrm{~Hz}, 1 \mathrm{H}, 4-\mathrm{H}), 8.23$ (broad dd, $\left.J=8.5 \mathrm{~Hz}, J^{\prime}=1.5 \mathrm{~Hz}, 1 \mathrm{H}, 1-\mathrm{H}\right) ;{ }^{13} \mathrm{C}$ NMR $(75.4 \mathrm{MHz}$, $\left.\mathrm{CDCl}_{3}\right) \delta 12.0\left(\mathrm{CH}_{3}, \mathrm{CH}_{2} \mathrm{CH}_{3}\right), 28.3(\mathrm{CH}, \mathrm{C} 7), 28.9\left(\mathrm{CH}_{2}, \mathrm{C} 13\right), 29.8\left(\mathrm{CH}_{2}, \mathrm{CH}_{2} \mathrm{CH}_{3}\right), 30.7$ 
(CH, C11), 35.6 ( $\left.\mathrm{CH}_{2}, \mathrm{C} 10\right), 40.5$ ( $\left.\mathrm{CH}_{2}, \mathrm{C} 6\right), 122.9$ (CH, C8), 123.9 (CH, C1), 125.4 (C) and 133.2 (C) (C11a and C12a), 126.3 (CH, C2), 128.5 (CH, C4), 129.2 (CH, C3), 138.0 (C), 140.7 (C), and 147.0 (C) (C4a, C5a, and C9), 158.8 (C, C12). Anal. ( $\mathrm{C}_{18} \mathrm{H}_{18} \mathrm{ClN}$ ) C, H, $\mathrm{N}, \mathrm{Cl}$.

\section{9-Ethyl-6,7,10,11-tetrahydro-12- $\{\{$ 8-[(1,2,3,4-tetrahydroacridin-9-} yl)amino]octyl $\}$ amino $\}$-7,11-methanocycloocta[b]quinoline dihydrochloride $\quad[( \pm)$ 12·2HCl]. A solution of chloroquinoline $( \pm)-\mathbf{8}(137 \mathrm{mg}, 0.48 \mathrm{mmol})$ and amine $\mathbf{1 1}^{9}$ (270 mg, 0.83 mmol, 1.7 equiv.) in 1-pentanol (2 mL) was heated under reflux for $42 \mathrm{~h}$. The mixture was cooled to room temperature, diluted with $\mathrm{CH}_{2} \mathrm{Cl}_{2}(30 \mathrm{~mL})$, and washed successively with $10 \%$ aqueous $\mathrm{NaOH}(30 \mathrm{~mL})$ and water $(2 \times 20 \mathrm{~mL})$. The organic layer was separated, dried with anhydrous $\mathrm{Na}_{2} \mathrm{SO}_{4}$, and evaporated at reduced pressure to give a yellow oil (425 mg). Column chromatography of this residue (70-200 mesh silica gel, $50 \mathrm{~g}$, $\mathrm{CH}_{2} \mathrm{Cl}_{2} / \mathrm{MeOH} / 25 \%$ aqueous $\mathrm{NH}_{4} \mathrm{OH}$ 70:30:0.7) afforded heterodimer ( \pm )-12 (58 mg, $21 \%$ yield) as a yellowish oil.

Treatment of a solution of $( \pm)$-12 (80 mg from another batch, $0.14 \mathrm{mmol})$ in $\mathrm{MeOH}(2 \mathrm{~mL})$ with a solution of $\mathrm{HCl}(1.81 \mathrm{~N}$ solution in $\mathrm{MeOH}, 0.5 \mathrm{~mL}$, 6.5 equiv.), followed by evaporation at reduced pressure, and washings with hot hexane $(3 \times 2 \mathrm{~mL})$ and AcOEt $(3 \times 2$ $\mathrm{mL}$ ), afforded pure $\mathbf{1 2} \cdot 2 \mathrm{HCl}(73 \mathrm{mg}$ ) as a light orange solid. The analytical sample of 12·2HCl (16 mg) was obtained by recrystallization from acetonitrile / hexane /AcOEt 3:1:1 as a light yellow solid: $\mathrm{mp} 199-200{ }^{\circ} \mathrm{C}$ (acetonitrile / hexane /AcOEt 3:1:1); $R_{f}[( \pm)-\mathbf{1 2}$ free 
base] $0.15\left(\mathrm{SiO}_{2}, \mathrm{CH}_{2} \mathrm{Cl}_{2} / \mathrm{MeOH} / 25 \%\right.$ aqueous $\mathrm{NH}_{4} \mathrm{OH}$ 9:1:0.07); IR (KBr) v3500-2500 (max. at 3421, 2926 and 2856, N+ $-\mathrm{H}, \mathrm{N}-\mathrm{H}$ and C-H st), 1636, 1587, and 1522 (ar-C-C and ar-C-N st) $\mathrm{cm}^{-1}$; ${ }^{1} \mathrm{H}$ NMR $\left(400 \mathrm{MHz}, \mathrm{CD}_{3} \mathrm{OD}\right) \delta 0.87$ (t, J=7.2 Hz, 3H, $\mathrm{CH}_{2} \mathrm{CH}_{3}$ ), $1.20-1.50$ (complex signal, 8H, 3'- $\mathrm{H}_{2}, 4^{\prime}-\mathrm{H}_{2}, 5^{\prime}-\mathrm{H}_{2}$ and $6^{\prime}-\mathrm{H}_{2}$ ), $1.50-2.10$ (complex signal, $13 \mathrm{H}, 10-\mathrm{H}_{\text {endo }}, 13-\mathrm{H}_{\text {syn }}, 13-\mathrm{H}_{\text {anti }}, \mathrm{CH}_{2} \mathrm{CH}_{3}, 2^{\prime}-\mathrm{H}_{2}, 7^{\prime}-\mathrm{H}_{2}, 2 "-\mathrm{H}_{2}$ and 3"- $\mathrm{H}_{2}$ ), 2.58 (broad d, J=16.4 Hz, 1H, 10- $\mathrm{H}_{\text {exo }}$ ), 2.69 (m, 2H, 1"- $\mathrm{H}_{2}$ ), 2.79 (m, 1H, 7-H), 2.90 (broad d, J=17.2 Hz, 1H, 6- $\mathrm{H}_{\text {endo }}$ ), $3.02\left(\mathrm{~m}, 2 \mathrm{H}, 4 "-\mathrm{H}_{2}\right), 3.21\left(\mathrm{dm}, J=17.2 \mathrm{~Hz}, 1 \mathrm{H}, 6-\mathrm{H}_{\text {exo }}\right.$ ), $3.49(\mathrm{~m}, 1 \mathrm{H}, 11-\mathrm{H}), 3.90-4.05$ (complex signal, 4H, 1'- $\mathrm{H}_{2}$ and 8'- $\left.\mathrm{H}_{2}\right), 4.86$ (s, $\mathrm{NH}, \mathrm{NH}^{+}$and $\mathrm{H}_{2} \mathrm{O}$ ), 5.58 (m, 1H, 8-H), 7.52-7.61 (complex signal, 2H, 2-H and 7"-H), 7.73-7.88 (complex signal, 4H, 3-H, 4-H, 5"-H and 6"-H), 8.34-8.42 (complex signal, 2H, 1-H and 8"-H); ${ }^{13} \mathrm{C}$ NMR (100.6 MHz, $\left.\mathrm{CD}_{3} \mathrm{OD}\right) \delta 12.6\left(\mathrm{CH}_{3}, \mathrm{CH}_{2} \mathrm{CH}_{3}\right), 21.8\left(\mathrm{CH}_{2}, \mathrm{C} 3 "\right), 23.0$ ( $\left.\mathrm{CH}_{2}, \mathrm{C} 2 "\right), 24.9\left(\mathrm{CH}_{2}, \mathrm{C} 1 "\right), 27.3(\mathrm{CH}, \mathrm{C} 11), 27.6\left(\mathrm{CH}_{2}\right)$ and $27.7\left(\mathrm{CH}_{2}\right)\left(\mathrm{C}^{\prime}\right.$ and $\left.\mathrm{C6}\right)$, $\left.27.8(\mathrm{CH}, \mathrm{C} 7), 29.3\left(\mathrm{CH}_{2}, \mathrm{C} 4\right)\right), 29.6\left(\mathrm{CH}_{2}, \mathrm{C} 13\right), 30.15\left(\mathrm{CH}_{2}\right)$ and $30.19\left(\mathrm{CH}_{2}\right)(\mathrm{C} 4$ ' and C5'), $30.9\left(\mathrm{CH}_{2}, \mathrm{CH}_{2} \mathrm{CH}_{3}\right), 31.4\left(\mathrm{CH}_{2}\right)$ and $31.5\left(\mathrm{CH}_{2}\right)(\mathrm{C} 2 '$ and $\mathrm{C} 7 '), 34.6\left(\mathrm{CH}_{2}, \mathrm{C} 10\right), 36.1$ ( $\left.\mathrm{CH}_{2}, \mathrm{C} 6\right), 49.1\left(\mathrm{CH}_{2}\right)$ and $49.5\left(\mathrm{CH}_{2}\right)\left(\mathrm{C}^{\prime}\right.$ and $\left.\mathrm{C} 8 '\right), 112.8$ (C, $\left.\mathrm{C} 9 " \mathrm{a}\right), 117.0(\mathrm{C}), 117.1(\mathrm{C})$ and 117.2 (C) (C11a, C12a and C8"a), 120.1 (2 CH, C4 and C5"), 123.4 (CH, C8), 126.2 $(\mathrm{CH}), 126.3(\mathrm{CH}), 126.5(\mathrm{CH})$, and $127.2(\mathrm{CH})(\mathrm{C} 1, \mathrm{C} 2, \mathrm{C} 7 "$, and $\mathrm{C} 8 "), 134.1(\mathrm{CH})$ and 134.2 (CH) (C3 and C6"), 139.7 (C), 140.0 (C), and 140.2 (C) (C4a , C9, and C10"a), 150.8 (C) and 151.7 (C) (C5a and C4"a), 157.0 (C) and 157.9 (C) (C12 and C9"). The signals of crystallization AcOEt were also observed in the ${ }^{1} \mathrm{H}$ and ${ }^{13} \mathrm{C}$ NMR spectra. Anal. $\left(\mathrm{C}_{39} \mathrm{H}_{48} \mathrm{~N}_{4} \cdot 2 \mathrm{HCl} \cdot 2 \mathrm{H}_{2} \mathrm{O} \cdot 0.5 \mathrm{AcOEt}\right) \mathrm{C}, \mathrm{H}, \mathrm{N}$. 
General Procedure for the Synthesis of Compounds 15 and 16. A mixture of finely powdered KOH (1.6 mmol), a solution of tacrine (1) or 6-chlorotacrine $(\mathbf{1 3})^{12}(1.0 \mathrm{mmol})$ in dry DMSO (1-4.5 mL) (previously prepared by heating at $70{ }^{\circ} \mathrm{C}$ in a water bath), and $4 \AA$ molecular sieves (approximately $100 \mathrm{mg}$ ) was thoroughly stirred at room temperature for 2 h. To the resulting mixture the dihaloderivative $\mathbf{1 4}(1.2 \mathrm{mmol})$ was added, and the reaction mixture was vigorously stirred at room temperature for $15 \mathrm{~h}$. Then, it was diluted with water $(20 \mathrm{~mL})$ and extracted with AcOEt $(4 \times 10 \mathrm{~mL})$. The combined organic extracts were washed with water $(3 \times 10 \mathrm{~mL})$, dried with anhydrous $\mathrm{Na}_{2} \mathrm{SO}_{4}$ and evaporated at reduced pressure to give a brown oily residue, which was submitted to column chromatography (70-200 mesh silica gel for 15a-c and 16a,c or 35-70 mesh silica gel for 15d and 16b,d, hexane / AcOEt mixtures for $\mathbf{1 5 a}-\mathbf{c}$ and $\mathbf{1 6 c}, \mathrm{CH}_{2} \mathrm{Cl}_{2} / \mathrm{MeOH}$ mixtures for 16a,b,d, and $\mathrm{CH}_{2} \mathrm{Cl}_{2} / \mathrm{MeOH} / 25 \%$ aqueous $\mathrm{NH}_{4} \mathrm{OH}$ mixtures for $\mathbf{1 5 d}$ ), to give compound $\mathbf{1 5}$ or $\mathbf{1 6}$ in most cases slightly impurified with the $\beta$-elimination by-product.

For characterization purposes the new compounds $15 \mathbf{d}$ and $16 \mathbf{d}$ were transformed into the corresponding dihydrochlorides by treatment with a $0.43 \mathrm{~N}$ solution of $\mathrm{HCl}$ in $\mathrm{MeOH}$ (6 equiv.), while the new compounds $\mathbf{1 6 a - c}$ were transformed into the corresponding hydrobromides by treatment with $48 \%$ aqueous $\mathrm{HBr}$ ( $2-3$ equiv.), and these salts were recrystallized and dried at $80^{\circ} \mathrm{C} / 1$ Torr for 2 days. 
9-[(6-Bromohexyl)amino]-1,2,3,4-tetrahydroacridine (15a): $:^{20,21} 1.70 \mathrm{~g}, 31 \%$ yield, yellowish oil: $R_{f} 0.27\left(\mathrm{SiO}_{2}\right.$, AcOEt / $\left.\mathrm{MeOH} 8: 2\right)$.

9-[(7-Bromoheptyl)amino]-1,2,3,4-tetrahydroacridine $\quad(\mathbf{1 5 b}):^{20} \quad 2.12$ g, 37\% yield, yellowish oil: $R_{f} 0.19\left(\mathrm{SiO}_{2}\right.$, AcOEt / $\left.\mathrm{MeOH} 8: 2\right)$.

9-[(8-Bromooctyl)amino]-1,2,3,4-tetrahydroacridine $\quad(\mathbf{1 5 c}):^{5} 565 \mathrm{mg}, \quad 29 \%$ yield, yellowish oil: $R_{f} 0.30\left(\mathrm{SiO}_{2}\right.$, AcOEt / $\left.\mathrm{MeOH} 8: 2\right)$.

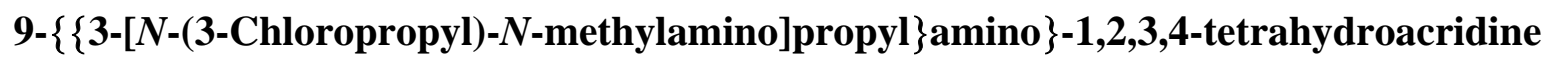
(15d): $793 \mathrm{mg}, 28 \%$ yield, yellowish oil: $R_{f} 0.50\left(\mathrm{SiO}_{2}, \mathrm{CH}_{2} \mathrm{Cl}_{2} / \mathrm{MeOH} / 25 \%\right.$ aqueous $\mathrm{NH}_{4} \mathrm{OH}$ 9:1:0.05). 15d·2HCl: light brown solid: mp 154-155 ${ }^{\circ} \mathrm{C}(\mathrm{MeOH} /$ AcOEt 1:20); IR (KBr) $v$ 3600-2500 (max. at 3404, 3065, 2942, 2861 and 2718, $\mathrm{N}^{+}-\mathrm{H}, \mathrm{N}-\mathrm{H}$ and C-H st), 1631, 1588 and 1522 (ar-C-C and ar-C-N st) cm ${ }^{-1} ;{ }^{1} \mathrm{H}$ NMR (300 MHz, CD $30 D$ ) $\delta 1.98$ (complex signal, 4H, 2- $\mathrm{H}_{2}$ and 3- $\mathrm{H}_{2}$ ), 2.20-2.38 (complex signal, 4H, 2'- $\mathrm{H}_{2}$ and 2"- $\mathrm{H}_{2}$ ), 2.78 (m, 2H, 1- $\left.\mathrm{H}_{2}\right), 2.91$ (s, 3H, N-CH ), 3.05 (m, 2H, 4- $\mathrm{H}_{2}$ ), 3.26-3.40 (complex signal, 4H, 3'- $\mathrm{H}_{2}$ and 1"- $\left.\mathrm{H}_{2}\right), 3.70\left(\mathrm{t}, J=6.3 \mathrm{~Hz}, 2 \mathrm{H}, 3 "-\mathrm{H}_{2}\right), 4.09\left(\mathrm{t}, J=7.2 \mathrm{~Hz}, 2 \mathrm{H}, 1^{\prime}-\mathrm{H}_{2}\right), 7.64$ (ddd, $\left.J=8.4 \mathrm{~Hz}, J^{\prime}=6.9 \mathrm{~Hz}, J^{\prime \prime}=1.5 \mathrm{~Hz}, 1 \mathrm{H}, 7-\mathrm{H}\right), 7.80$ (dd, $J \approx 8.4 \mathrm{~Hz}, J^{\prime} \approx 1.5 \mathrm{~Hz}, 1 \mathrm{H}, 5-\mathrm{H}$ ), 7.88 (ddd, $J=8.4 \mathrm{~Hz}, J^{\prime}=6.9 \mathrm{~Hz}, J^{\prime \prime}=1.2 \mathrm{~Hz}, 1 \mathrm{H}, 6-\mathrm{H}$ ), 8.44 (broad d, $J=8.4 \mathrm{~Hz}, 1 \mathrm{H}, 8-\mathrm{H}$ );

${ }^{13} \mathrm{C}$ NMR (75.4 MHz, CD $\left.3 \mathrm{OD}\right) \delta 21.8\left(\mathrm{CH}_{2}, \mathrm{C} 3\right), 23.0\left(\mathrm{CH}_{2}, \mathrm{C} 2\right), 25.3\left(\mathrm{CH}_{2}, \mathrm{C} 1\right), 26.5$ (CH 
( $\mathrm{CH}_{2}, \mathrm{C} 1$ '), $54.7\left(\mathrm{CH}_{2}\right)$ and $55.1\left(\mathrm{CH}_{2}\right)\left(\mathrm{C}^{\prime}\right.$ and $\left.\mathrm{C} 1 "\right), 113.5$ (C, $\left.\mathrm{C} 9 \mathrm{a}\right), 117.2$ (C, C8a), 120.1 (CH, C5), 126.2 (CH, C7), 126.7 (CH, C8), 134.1 (CH, C6), 139.6 (C, C10a), 152.2 (C, C4a), 157.9 (C, C9). Anal. $\left(\mathrm{C}_{20} \mathrm{H}_{28} \mathrm{ClN}_{3} \cdot 2 \mathrm{HCl} \cdot \mathrm{H}_{2} \mathrm{O} \cdot 0.2 \mathrm{AcOEt}\right) \mathrm{C}, \mathrm{H}, \mathrm{N}, \mathrm{Cl}$.

9-[(6-Bromohexyl)amino]-6-chloro-1,2,3,4-tetrahydroacridine (16a): 1.06 g, 31\% yield, yellowish oil: $R_{f} 0.69\left(\mathrm{SiO}_{2}, \mathrm{CH}_{2} \mathrm{Cl}_{2} / \mathrm{MeOH} / 25 \%\right.$ aqueous $\mathrm{NH}_{4} \mathrm{OH}$ 9:1:0.05). 16a·HBr: light yellow solid: mp 85-86 ${ }^{\circ} \mathrm{C}(\mathrm{MeOH} /$ AcOEt 1:20); IR (KBr) v 3500-2500 (max. at 3415, 3250, 3043, 2999, 2933, 2856 and 2790, N+ $-\mathrm{H}, \mathrm{N}-\mathrm{H}$ and C-H st), 1628, 1571, 1522 and 1513 (ar-C-C and ar-C-N st) cm ${ }^{-1}$; ${ }^{1} \mathrm{H}$ NMR (300 MHz, CD ${ }_{3} \mathrm{OD}$ ) $\delta 1.42-1.54$ (complex signal, $4 \mathrm{H}, 3^{\prime}-\mathrm{H}_{2}$ and $4^{\prime}-\mathrm{H}_{2}$ ), 1.80-2.02 (complex signal, $8 \mathrm{H}, 2-\mathrm{H}_{2}, 3-\mathrm{H}_{2}, 2^{\prime}-\mathrm{H}_{2}$ and 5'- $\mathrm{H}_{2}$ ), 2.69 (m, 2H, 1- $\mathrm{H}_{2}$ ), 3.00 (m, 2H, 4- $\mathrm{H}_{2}$ ), 3.44 (t, $\left.J=6.6 \mathrm{~Hz}, 2 \mathrm{H}, 6^{\prime}-\mathrm{H}_{2}\right), 3.96$ (tm, $J=7.2 \mathrm{~Hz}$, 2H 1'- $\mathrm{H}_{2}$ ), 4.85 (s, NH and $\mathrm{NH}^{+}$), $7.56\left(\mathrm{dd}, J=9.3 \mathrm{~Hz}, J^{\prime}=2.4 \mathrm{~Hz}, 1 \mathrm{H}, 7-\mathrm{H}\right), 7.78$ (d, $J \approx 2.4$ Hz, 1H, 5-H), 8.39 (d, J=9.3 Hz, 1H, 8-H); ${ }^{13} \mathrm{C}$ NMR (75.4 MHz, CD $\left.{ }_{3} \mathrm{OD}\right) \delta 21.8\left(\mathrm{CH}_{2}\right.$, C3), $22.9\left(\mathrm{CH}_{2}, \mathrm{C} 2\right), 24.8\left(\mathrm{CH}_{2}, \mathrm{C} 1\right), 26.8\left(\mathrm{CH}_{2}, \mathrm{C} 3\right), 28.7\left(\mathrm{CH}_{2}, \mathrm{C} 4\right), 29.3\left(\mathrm{CH}_{2}, \mathrm{C} 4\right)$, $31.1\left(\mathrm{CH}_{2}\right)$ and $33.7\left(\mathrm{CH}_{2}\right)\left(\mathrm{C}^{\prime}\right.$ and $\left.\mathrm{C}^{\prime}\right), 34.3\left(\mathrm{CH}_{2}, \mathrm{C}^{\prime}\right), 49.1\left(\mathrm{CH}_{2}, \mathrm{C} 1{ }^{\prime}\right), 113.3(\mathrm{C})$ and 115.4 (C) (C8a and C9a), 119.0 (CH, C5), 126.7 (CH, C7), 128.7 (CH, C8), 140.0 (C, C6), 140.4 (C, C10a), 152.0 (C, C4a), 157.8 (C, C9). HRMS calcd for $\mathrm{C}_{19} \mathrm{H}_{24} \mathrm{ClBrN}_{2}: 394.0811$. Found: 394.0810 .

9-[(7-Bromoheptyl)amino]-6-chloro-1,2,3,4-tetrahydroacridine (16b): 1.56 g, 44\% yield, yellowish oil: $R_{f} 0.80\left(\mathrm{SiO}_{2}, \mathrm{CH}_{2} \mathrm{Cl}_{2} / \mathrm{MeOH} / 25 \%\right.$ aqueous $\mathrm{NH}_{4} \mathrm{OH}$ 9:1:0.05). 
16b·HBr: light orange-colored solid: $\mathrm{mp} 78-79{ }^{\circ} \mathrm{C}(\mathrm{MeOH} / \mathrm{AcOEt} 1: 10)$; $\mathrm{IR}(\mathrm{KBr}) v$ 3500-2500 (max. at 3404, 3248, 3043, 2930, 2856 and 2790, N $-\mathrm{H}, \mathrm{N}-\mathrm{H}$ and C-H st), 1628, 1571 and 1522 (ar-C-C and ar-C-N st) cm ${ }^{-1} ;{ }^{1} \mathrm{H}$ NMR (300 MHz, CD ${ }_{3} \mathrm{OD}$ ) $\delta$ 1.24-1.52 (complex signal, 6H, 3'- $\mathrm{H}_{2}, 4^{\prime}-\mathrm{H}_{2}$ and 5'- $\mathrm{H}_{2}$ ), 1.64-2.04 (complex signal, 8H, 2$\mathrm{H}_{2}, 3-\mathrm{H}_{2}, 2^{\prime}-\mathrm{H}_{2}$ and 6'- $\left.\mathrm{H}_{2}\right), 2.68\left(\mathrm{~m}, 2 \mathrm{H}, 1-\mathrm{H}_{2}\right), 3.00\left(\mathrm{~m}, 2 \mathrm{H}, 4-\mathrm{H}_{2}\right), 3.43(\mathrm{t}, J=6.6 \mathrm{~Hz}, 2 \mathrm{H}$, 7'- $\mathrm{H}_{2}$ ), 3.95 (t, $\left.J=7.2 \mathrm{~Hz}, 2 \mathrm{H}, 1^{\prime}-\mathrm{H}_{2}\right), 4.85\left(\mathrm{~s}, \mathrm{NH}\right.$ and $\mathrm{NH}^{+}$), 7.57 (dd, $J=9.3 \mathrm{~Hz}, J^{\prime}=2.1 \mathrm{~Hz}$, 1H, 7-H), 7.77 (d, $J=2.1 \mathrm{~Hz}, 1 \mathrm{H}, 5-\mathrm{H}), 8.39$ (d, $J=9.3 \mathrm{~Hz}, 1 \mathrm{H}, 8-\mathrm{H}) ;{ }^{13} \mathrm{C}$ NMR $(75.4 \mathrm{MHz}$, $\left.\mathrm{CD}_{3} \mathrm{OD}\right) \delta 21.8\left(\mathrm{CH}_{2}, \mathrm{C} 3\right), 22.9\left(\mathrm{CH}_{2}, \mathrm{C} 2\right), 24.8\left(\mathrm{CH}_{2}, \mathrm{C} 1\right), 27.5\left(\mathrm{CH}_{2}, \mathrm{C} 3\right), 28.9\left(\mathrm{CH}_{2}\right.$, C5'), $29.30\left(\mathrm{CH}_{2}\right)$ and $29.34\left(\mathrm{CH}_{2}\right)\left(\mathrm{C} 4\right.$ and $\left.\mathrm{C}^{\prime}\right), 31.2\left(\mathrm{CH}_{2}\right)$ and $33.8\left(\mathrm{CH}_{2}\right)\left(\mathrm{C}^{\prime}\right.$ and $\left.\mathrm{C}^{\prime}\right)$, $34.5\left(\mathrm{CH}_{2}, \mathrm{C}^{\prime}\right), 49.1\left(\mathrm{CH}_{2}, \mathrm{C1}\right), 113.3$ (C) and 115.3 (C) (C8a and $\left.\mathrm{C} 9 \mathrm{a}\right), 119.0$ (CH, C5), 126.7 (CH, C7), 128.7 (CH, C8), 140.0 (C, C6), 140.3 (C, C10a), 152.0 (C, C4a), 157.7 (C, C9). HRMS calcd for $\mathrm{C}_{20} \mathrm{H}_{26} \mathrm{ClBrN}_{2}$ : 408.0968. Found: 408.0970 .

9-[(8-Bromooctyl)amino]-6-chloro-1,2,3,4-tetrahydroacridine (16c): 559 mg, 31\% yield, yellowish oil: $R_{f} 0.37\left(\mathrm{SiO}_{2}\right.$, AcOEt / $\left.\mathrm{MeOH} 4: 1\right)$. 16c·HBr: light yellow solid: mp 60-61 ${ }^{\circ} \mathrm{C}(\mathrm{MeOH}) ; \mathrm{IR}(\mathrm{KBr}) v 3500-2500$ (max. at 3404, 3252, 3013, 2929, 2855 and 2790, $\mathrm{N}^{+}-$ H, N-H and C-H st), 1629, 1572 and 1522 (ar-C-C and ar-C-N st) $\mathrm{cm}^{-1}$; ${ }^{1} \mathrm{H}$ NMR (400 $\mathrm{MHz}, \mathrm{CD}_{3} \mathrm{OD}$ ) $\delta 1.30-1.48$ (complex signal, $8 \mathrm{H}, 3^{\prime}-\mathrm{H}_{2}, 4^{\prime}-\mathrm{H}_{2}, 5^{\prime}-\mathrm{H}_{2}$ and $6^{\prime}-\mathrm{H}_{2}$ ), 1.72-1.88 (complex signal, 4H, 2'- $\mathrm{H}_{2}$ and 7'- $\mathrm{H}_{2}$ ), 1.90-2.02 (complex signal, 4H, 2- $\mathrm{H}_{2}$ and 3- $\mathrm{H}_{2}$ ), 2.68 (m, 2H, 1- $\left.\mathrm{H}_{2}\right), 3.00\left(\mathrm{~m}, 2 \mathrm{H}, 4-\mathrm{H}_{2}\right), 3.43$ (t, $\left.J=6.6 \mathrm{~Hz}, 2 \mathrm{H}, 8^{\prime}-\mathrm{H}_{2}\right), 3.95$ (t, $J=7.2 \mathrm{~Hz}, 2 \mathrm{H}$, 1'- $\mathrm{H}_{2}$ ), 4.86 (s, NH and $\mathrm{NH}^{+}$), $7.56\left(\mathrm{dd}, J=9.2 \mathrm{~Hz}, J^{\prime}=2.0 \mathrm{~Hz}, 1 \mathrm{H}, 7-\mathrm{H}\right), 7.78$ (d, $J \approx 2.0 \mathrm{~Hz}$, 


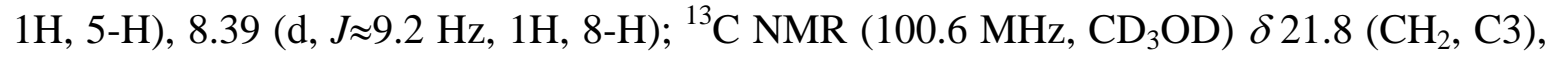
$22.8\left(\mathrm{CH}_{2}, \mathrm{C} 2\right), 24.7\left(\mathrm{CH}_{2}, \mathrm{C} 1\right), 27.5\left(\mathrm{CH}_{2}, \mathrm{C} 3\right), 28.9\left(\mathrm{CH}_{2}, \mathrm{C} 5\right), 29.3\left(\mathrm{CH}_{2}, \mathrm{C} 4\right), 29.6$ $\left(\mathrm{CH}_{2}\right)$ and $30.0\left(\mathrm{CH}_{2}\right)\left(\mathrm{C}^{\prime}\right.$ and $\left.\mathrm{C}^{\prime}\right), 31.3\left(\mathrm{CH}_{2}\right)$ and $33.8\left(\mathrm{CH}_{2}\right)\left(\mathrm{C}^{\prime}\right.$ and $\left.\mathrm{C} 8 '\right), 34.5\left(\mathrm{CH}_{2}\right.$, C7'), 49.2 ( $\left.\mathrm{CH}_{2}, \mathrm{C}^{\prime}\right), 113.4$ (C) and 115.4 (C) (C8a and C9a), 119.1 (CH, C5), 126.8 (CH, C7), 128.8 (CH, C8), 140.1 (C, C6), 140.5 (C, C10a), 152.1 (C, C4a), 157.9 (C, C9). HRMS calcd for $\mathrm{C}_{21} \mathrm{H}_{28} \mathrm{ClBrN}_{2}$ : 422.1124. Found: 422.1123.

\section{6-Chloro-9- $\{\{3-[N-(3-C h l o r o p r o p y l)-N$-methylamino]propyl $\}$ amino $\}-1,2,3,4-$}

tetrahydroacridine (16d): $646 \mathrm{mg}, 20 \%$ yield, yellowish oil: $R_{f} 0.64\left(\mathrm{SiO}_{2}, \mathrm{CH}_{2} \mathrm{Cl}_{2} /\right.$ $\mathrm{MeOH} / 25 \%$ aqueous $\mathrm{NH}_{4} \mathrm{OH}$ 9:1:0.05). 16d·2HCl: light brown solid: mp 181-182 ${ }^{\circ} \mathrm{C}$ $\left(\mathrm{CH}_{2} \mathrm{Cl}_{2}\right)$; IR (KBr) v 3600-2500 (max. at 3410, 3255, 3126, 3051, 2945, 2868, 2793 and 2707, $\mathrm{N}^{+}-\mathrm{H}, \mathrm{N}-\mathrm{H}$ and C-H st), 1630, 1585 and 1513 (ar-C-C and ar-C-N st) $\mathrm{cm}^{-1}$; ${ }^{1} \mathrm{H}$ NMR (300 MHz, $\mathrm{CD}_{3} \mathrm{OD}$ ) $\delta 1.97$ (complex signal, 4H, 2- $\mathrm{H}_{2}$ and 3- $\mathrm{H}_{2}$ ), 2.20-2.40 (complex signal, 4H, 2'- $\mathrm{H}_{2}$ and 2"- $\left.\mathrm{H}_{2}\right), 2.76\left(\mathrm{~m}, 2 \mathrm{H}, 1-\mathrm{H}_{2}\right), 2.93\left(\mathrm{~s}, 3 \mathrm{H}, \mathrm{N}-\mathrm{CH}_{3}\right), 3.04\left(\mathrm{~m}, 2 \mathrm{H}, 4-\mathrm{H}_{2}\right)$, 3.22-3.48 (complex signal, 4H, 3'- $\mathrm{H}_{2}$ and 1"- $\mathrm{H}_{2}$ ), $3.72\left(\mathrm{t}, \mathrm{J}=6.3 \mathrm{~Hz}, 2 \mathrm{H}, 3 "-\mathrm{H}_{2}\right), 4.09$ (t, $J=7.2 \mathrm{~Hz}, 2 \mathrm{H}, 1^{\prime}-\mathrm{H}_{2}$ ), 4.88 (s, NH and $\mathrm{NH}^{+}$), 7.59 (dd, $\left.J=9.3 \mathrm{~Hz}, J^{\prime}=2.1 \mathrm{~Hz}, 1 \mathrm{H}, 7-\mathrm{H}\right), 7.82$

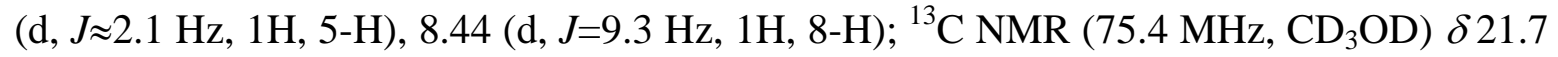
( $\left.\left.\mathrm{CH}_{2}, \mathrm{C} 3\right), 22.9\left(\mathrm{CH}_{2}, \mathrm{C} 2\right), 25.2\left(\mathrm{CH}_{2}, \mathrm{C} 1\right), 26.3\left(\mathrm{CH}_{2}, \mathrm{C} 2\right), 28.2\left(\mathrm{CH}_{2}, \mathrm{C} 2\right)\right), 29.5\left(\mathrm{CH}_{2}\right.$, C4), $40.7\left(\mathrm{CH}_{3}, \mathrm{~N}-\mathrm{CH}_{3}\right), 42.3\left(\mathrm{CH}_{2}, \mathrm{C} 3 "\right), 45.9\left(\mathrm{CH}_{2}, \mathrm{C1}\right), 54.6\left(\mathrm{CH}_{2}\right)$ and $55.1\left(\mathrm{CH}_{2}\right)\left(\mathrm{C}^{\prime}\right.$ and C1"), 113.9 (C) and 115.6 (C) (C8a and C9a), 119.1 (CH, C5), 127.1 (CH, C7), 128.6 
(CH, C8), 140.0 (C, C6), 140.2 (C, C10a), 152.6 (C, C4a), 157.7 (C, C9). HRMS calcd for $\mathrm{C}_{20} \mathrm{H}_{27} \mathrm{Cl}_{2} \mathrm{~N}_{3}$ : 379.1582. Found: 379.1589.

General Procedure for the Synthesis of Heterodimers $( \pm)-17$ and $( \pm)-18$. These compounds were prepared as previously described for compounds 15 and 16, from a mixture of finely powdered $\mathrm{KOH}(2.6 \mathrm{mmol})$, a solution of $( \pm)$-huprine $\mathrm{Y}[( \pm)-4](1.0$ mmol) in dry DMSO (4.5 mL) (previously prepared by heating at $70^{\circ} \mathrm{C}$ in a water bath), 4 $\AA$ molecular sieves (approximately $400 \mathrm{mg}$ ), and a solution of compound $\mathbf{1 5}$ or $\mathbf{1 6}$ (1.2 mmol) in dry DMSO (1.5 mL), with longer reaction times (54-86 h). The resulting brown oily residue was submitted to column chromatography (35-70 mesh silica gel, $\mathrm{CH}_{2} \mathrm{Cl}_{2}$ / $\mathrm{MeOH}$ mixtures for $\mathbf{1 7 a}-\mathbf{c}$ and $\mathbf{1 8 a}-\mathbf{c}$ and $\mathrm{CH}_{2} \mathrm{Cl}_{2} / \mathrm{MeOH} / 25 \%$ aqueous $\mathrm{NH}_{4} \mathrm{OH}$ mixtures for 17d and 18d), to give pure heterodimers $( \pm)-\mathbf{1 7}$ or $( \pm)$-18 as light yellow oils, which were transformed into the corresponding di- or tri-hydrochlorides by treatment with a $0.48 \mathrm{~N}$ solution of $\mathrm{HCl}$ in $\mathrm{MeOH}$ (6-9 equiv.), followed by evaporation, recrystallization from $\mathrm{MeOH} / \mathrm{AcOEt}$ mixtures, and drying at $80^{\circ} \mathrm{C} / 1$ Torr for 2 days.

\section{3-Chloro-6,7,10,11-tetrahydro-9-methyl-12-\{\{6-[(1,2,3,4-tetrahydroacridin-9-}

yl)amino]hexyl \}amino $\}$-7,11-methanocycloocta[b]quinoline dihydrochloride $[( \pm)$ -

17a·2HCl]: $214 \mathrm{mg}, 23 \%$ yield (free base), yellowish oil: $R_{f} 0.41\left(\mathrm{SiO}_{2}, \mathrm{CH}_{2} \mathrm{Cl}_{2} / \mathrm{MeOH} /\right.$ 25\% aqueous $\mathrm{NH}_{4} \mathrm{OH}$ 9:1:0.05). ( \pm )-17a·2HCl: light yellow solid: mp 252-253 ${ }^{\circ} \mathrm{C}(\mathrm{MeOH}$ / AcOEt 1:23); IR (KBr) v3600-2500 (max. at 3421, 3249, 3060, 2930 and 2860, N+ $-\mathrm{H}, \mathrm{N}-$ 
$\mathrm{H}$ and C-H st), 1630, 1582 and 1522 (ar-C-C and ar-C-N st) cm ${ }^{-1}$; ${ }^{1} \mathrm{H}$ NMR (400 MHz, $\mathrm{CD}_{3} \mathrm{OD}$ ) $\delta 1.53$ (complex signal, 4H, 3'- $\mathrm{H}_{2}$ and $4 '-\mathrm{H}_{2}$ ), 1.56 (s, 3H, 9- $\mathrm{CH}_{3}$ ), $1.84-2.00$

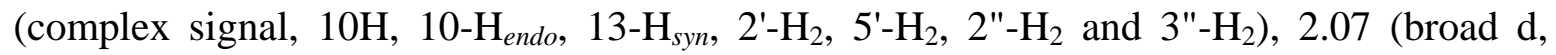
$\left.J=10.8 \mathrm{~Hz}, 1 \mathrm{H}, 13-\mathrm{H}_{\text {anti }}\right), 2.55$ (broad d, $J=16.8 \mathrm{~Hz}, 1 \mathrm{H}, 10-\mathrm{H}_{\text {exo }}$ ), $2.71\left(\mathrm{~m}, 2 \mathrm{H}, 1 "-\mathrm{H}_{2}\right), 2.76$ (m, 1H, 7-H), 2.88 (d, J=17.6 Hz, 1H, 6- $\mathrm{H}_{\text {endo }}$ ), 3.02 (m, 2H, 4"-- $\mathrm{H}_{2}$ ), 3.20 (dd, J=17.6 Hz, $\left.J^{\prime}=4.8 \mathrm{~Hz}, 1 \mathrm{H}, 6-\mathrm{H}_{\text {exo }}\right), 3.47(\mathrm{~m}, 1 \mathrm{H}, 11-\mathrm{H}), 3.98$ (complex signal, 4H, 1'- $\mathrm{H}_{2}$ and 6'- $\mathrm{H}_{2}$ ), 4.85 (s, $\mathrm{NH}, \mathrm{NH}^{+}$and $\mathrm{H}_{2} \mathrm{O}$ ), 5.58 (broad d, $J=4.0 \mathrm{~Hz}, 1 \mathrm{H}, 8-\mathrm{H}$ ), 7.52 (broad d, $J=8.8 \mathrm{~Hz}$, 1H, 2-H), 7.56 (dd, $J=J^{\prime}=7.6 \mathrm{~Hz}, 1 \mathrm{H}, 7$ "-H), 7.77 (broad s, 1H, 4-H), superimposed in part 7.78 (d, J=6.0 Hz, 1H, 5"-H), 7.84 (m, 1H, 6"-H), 8.36-8.42 (complex signal, 2H, 1-H and 8"-H); ${ }^{13} \mathrm{C}$ NMR (100.6 MHz, $\left.\mathrm{CD}_{3} \mathrm{OD}\right) \delta 21.8\left(\mathrm{CH}_{2}, \mathrm{C} 3 "\right), 23.0\left(\mathrm{CH}_{2}, \mathrm{C} 2 "\right), 23.5\left(\mathrm{CH}_{3}\right.$, 9CH $\left.\mathrm{CH}_{3}\right), 25.0\left(\mathrm{CH}_{2}, \mathrm{C} 1 "\right), 27.3(\mathrm{CH}, \mathrm{C} 11), 27.4\left(\mathrm{CH}_{2}\right)$ and $27.5\left(\mathrm{CH}_{2}\right)\left(\mathrm{C}^{\prime}\right.$ and $\left.\mathrm{C} 4\right), 27.8$ (CH, C7), $29.3\left(2 \mathrm{CH}_{2}, \mathrm{C} 13\right.$ and $\left.\mathrm{C} 4 "\right), 31.2\left(\mathrm{CH}_{2}\right)$ and $31.5\left(\mathrm{CH}_{2}\right)\left(\mathrm{C}^{\prime}\right.$ and $\left.\mathrm{C}^{\prime}\right), 36.1\left(\mathrm{CH}_{2}\right.$, C10), $36.2\left(\mathrm{CH}_{2}, \mathrm{C} 6\right), 48.6\left(\mathrm{CH}_{2}\right)$ and $49.5\left(\mathrm{CH}_{2}\right)\left(\mathrm{C}^{\prime}\right.$ and $\left.\mathrm{C}^{\prime}\right), 112.9(\mathrm{C}), 115.6(\mathrm{C}), 117.0$ (C), and 117.6 (C) (C11a, C12a, C8"a, and C9"a), $119.1(\mathrm{CH})$ and $120.1(\mathrm{CH})(\mathrm{C} 4$ and C5"), 125.2 (CH, C8), 126.4 (CH, C7"), 126.5 (CH, C8"), 126.7 (CH, C2), 129.5 (CH, C1), 134.1 (CH, C6"), 134.5 (C, C9), 139.7 (C), 140.2 (C) and 140.9 (C) (C3, C4a and C10"a), 151.2 (C) and 151.7 (C) (C5a and C4"a), 156.8 (C) and 157.9 (C) (C12 and C9"). Anal. $\left(\mathrm{C}_{36} \mathrm{H}_{41} \mathrm{ClN}_{4} \cdot 2.1 \mathrm{HCl} \cdot 3.5 \mathrm{H}_{2} \mathrm{O}\right) \mathrm{C}, \mathrm{H}, \mathrm{N}, \mathrm{Cl}$.

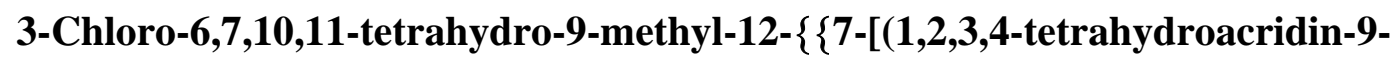
yl)amino]heptyl\}amino\}-7,11-methanocycloocta[b]quinoline dihydrochloride $[( \pm)$ - 
17b·2HCl]: 353 mg, 44\% yield (free base), light yellow oil: $R_{f} 0.48\left(\mathrm{SiO}_{2}, \mathrm{CH}_{2} \mathrm{Cl}_{2} / \mathrm{MeOH}\right.$ / 25\% aqueous $\mathrm{NH}_{4} \mathrm{OH}$ 9:1:0.05). ( \pm )-17b·2HCl: light brown solid: mp $239-240{ }^{\circ} \mathrm{C}$ (MeOH / AcOEt 2:7); IR (KBr) v 3500-2500 (max. at 3420, 3258, 3113, 3057, 2930 and 2855, N ${ }^{+}-\mathrm{H}, \mathrm{N}-\mathrm{H}$ and C-H st), 1629, 1583 and 1522 (ar-C-C and ar-C-N st) $\mathrm{cm}^{-1}$; ${ }^{1} \mathrm{H}$ NMR (400 MHz, $\mathrm{CD}_{3} \mathrm{OD}$ ) $\delta 1.47$ (complex signal, 6H, 3'- $-\mathrm{H}_{2}, 4^{\prime}-\mathrm{H}_{2}$ and 5'- $\mathrm{H}_{2}$ ), 1.57 (s, 3H, 9$\mathrm{CH}_{3}$ ), 1.80-2.00 (complex signal, $10 \mathrm{H}, 10-\mathrm{H}_{\text {endo }}, 13-\mathrm{H}_{\text {syn }}, 2^{\prime}-\mathrm{H}_{2}, 6$ 6'- $\mathrm{H}_{2}, 2^{\prime \prime}-\mathrm{H}_{2}$ and 3"- $\mathrm{H}_{2}$ ), 2.07 (dm, $J=12.4 \mathrm{~Hz}, 1 \mathrm{H}, 13-\mathrm{H}_{\text {anti }}$ ), 2.55 (broad dd, $J=17.6 \mathrm{~Hz}, J^{\prime}=3.6 \mathrm{~Hz}, 1 \mathrm{H}, 10-\mathrm{H}_{\text {exo }}$ ),

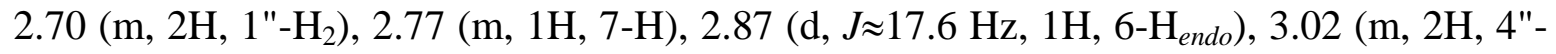
$\mathrm{H}_{2}$ ), 3.20 (dd, $\left.J=17.6 \mathrm{~Hz}, J^{\prime}=5.6 \mathrm{~Hz}, 1 \mathrm{H}, 6-\mathrm{H}_{\text {exo }}\right), 3.46(\mathrm{~m}, 1 \mathrm{H}, 11-\mathrm{H}), 3.96$ (t, $J=8.0 \mathrm{~Hz}$, $2 \mathrm{H})$ and $3.98(\mathrm{t}, \mathrm{J}=8.0 \mathrm{~Hz}, 2 \mathrm{H})\left(1^{\prime}-\mathrm{H}_{2}\right.$ and $\left.7^{\prime}-\mathrm{H}_{2}\right), 4.85$ (s, NH, $\mathrm{NH}^{+}$and $\mathrm{H}_{2} \mathrm{O}$ ), 5.58 (broad d, $J=4.8 \mathrm{~Hz}, 1 \mathrm{H}, 8-\mathrm{H}$ ), 7.53 (dd, $J=9.2 \mathrm{~Hz}, J^{\prime}=2.0 \mathrm{~Hz}, 1 \mathrm{H}, 2-\mathrm{H}$ ), 7.56 (broad dd, $J \approx J^{\prime} \approx 7.6$ Hz, 1H, 7"-H), 7.77 (broad s, 1H, 4-H), 7.78 (d, $J=6.0 \mathrm{~Hz}, 1 \mathrm{H}, 5$ "-H), 7.83 (dd, $J=J^{\prime}=7.6$ $\mathrm{Hz}, 1 \mathrm{H}, 6$ "-H), 8.36-8.40 (complex signal, 2H, 1-H and 8"-H); ${ }^{13} \mathrm{C}$ NMR (100.6 MHz, $\left.\mathrm{CD}_{3} \mathrm{OD}\right) \delta 21.8\left(\mathrm{CH}_{2}, \mathrm{C} 3 "\right), 23.0\left(\mathrm{CH}_{2}, \mathrm{C} 2 "\right), 23.5\left(\mathrm{CH}_{3}, 9-\mathrm{CH}_{3}\right), 24.9\left(\mathrm{CH}_{2}, \mathrm{C} 1 "\right), 27.3$ (CH, C11), $27.6\left(\mathrm{CH}_{2}\right)$ and $27.7\left(\mathrm{CH}_{2}\right)\left(\mathrm{C}^{\prime}\right.$ and $\left.\mathrm{C}^{\prime}\right), 27.9(\mathrm{CH}, \mathrm{C} 7), 29.3\left(2 \mathrm{CH}_{2}, \mathrm{C} 13\right.$ and C4"), $29.9\left(\mathrm{CH}_{2}, \mathrm{C}^{\prime}\right), 31.2\left(\mathrm{CH}_{2}\right)$ and $31.5\left(\mathrm{CH}_{2}\right)\left(\mathrm{C} 2\right.$ ' and $\left.\mathrm{C}^{\prime}\right), 36.06\left(\mathrm{CH}_{2}\right)$ and 36.13 ( $\mathrm{CH}_{2}$, C6 and C10), $49.1\left(\mathrm{CH}_{2}\right)$ and $49.6\left(\mathrm{CH}_{2}\right)\left(\mathrm{C}^{\prime}\right.$ and $\left.\mathrm{C}^{\prime}\right), 112.8(\mathrm{C}), 115.6(\mathrm{C}), 117.0$ (C), and 117.6 (C) (C11a, C12a, C8"a, and C9"a), 119.2 (CH) and 120.1 (CH) (C4 and C5"), 125.2 (CH, C8), 126.3 (CH, C7"), 126.5 (CH, C8"), 126.6 (CH, C2), 129.5 (CH, C1), 134.1 (CH, C6"), 134.5 (C, C9), 139.8 (C), 140.2 (C) and 141.0 (C) (C3, C4a and C10"a), 
151.3 (C) and 151.7 (C) (C5a and C4"a), 156.8 (C) and 157.9 (C) (C12 and C9"). Anal. $\left(\mathrm{C}_{37} \mathrm{H}_{43} \mathrm{ClN}_{4} \cdot 2 \mathrm{HCl} \cdot 3.2 \mathrm{H}_{2} \mathrm{O} \cdot 0.1 \mathrm{AcOEt}\right) \mathrm{C}, \mathrm{H}, \mathrm{N}, \mathrm{Cl}$.

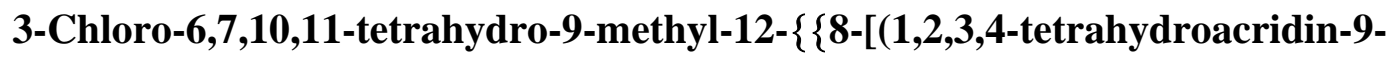

yl)amino]octyl $\}$ amino $\}$-7,11-methanocycloocta[b]quinoline dihydrochloride $\quad[( \pm)$ -

17c·2HCl]: 229 mg, 40\% yield (free base), light yellow oil: $R_{f} 0.39\left(\mathrm{SiO}_{2}, \mathrm{CH}_{2} \mathrm{Cl}_{2} / \mathrm{MeOH}\right.$ / 25\% aqueous $\mathrm{NH}_{4} \mathrm{OH}$ 9:1:0.05). ( \pm )-17c·2HCl: light brown solid: mp 233-234 ${ }^{\circ} \mathrm{C}(\mathrm{MeOH}$ / AcOEt 1:4); IR (KBr) v3500-2500 (max. at 3418, 3258, 3046, 2928, 2855 and 2800, $\mathrm{N}^{+}-$ H, N-H and C-H st), 1630, 1584 and 1523 (ar-C-C and ar-C-N st) $\mathrm{cm}^{-1}$; ${ }^{1} \mathrm{H}$ NMR (400 MHz, $\mathrm{CD}_{3} \mathrm{OD}$ ) $\delta 1.42$ (complex signal, 8H, 3'- $-\mathrm{H}_{2}, 4^{\prime}-\mathrm{H}_{2}, 5^{\prime}-\mathrm{H}_{2}$ and 6'- $\mathrm{H}_{2}$ ), 1.57 (s, 3H, 9$\mathrm{CH}_{3}$ ), 1.80-2.01 (complex signal, $10 \mathrm{H}, 10-\mathrm{H}_{\text {endo }}, 13-\mathrm{H}_{\text {syn }}, 2^{\prime}-\mathrm{H}_{2}, 7^{\prime}-\mathrm{H}_{2}, 2$ '-- $\mathrm{H}_{2}$ and 3"- $\mathrm{H}_{2}$ ), 2.08 (broad d, $J=12.0 \mathrm{~Hz}, 1 \mathrm{H}, 13-\mathrm{H}_{\text {anti }}$ ), 2.55 (broad d, $J=16.4 \mathrm{~Hz}, 1 \mathrm{H}, 10-\mathrm{H}_{\text {exo }}$ ), 2.71 (m,

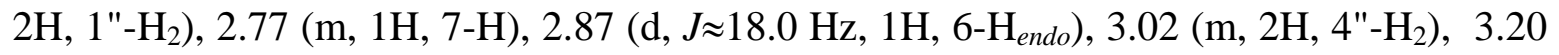
(dd, $J=18.0 \mathrm{~Hz}, J^{\prime}=5.2 \mathrm{~Hz}, 1 \mathrm{H}, 6-\mathrm{H}_{\text {exo }}$ ), $3.45(\mathrm{~m}, 1 \mathrm{H}, 11-\mathrm{H}), 3.95(\mathrm{t}, J=7.2 \mathrm{~Hz}, 2 \mathrm{H})$ and 3.97 (t, $J=7.2 \mathrm{~Hz}, 2 \mathrm{H})\left(1^{\prime}-\mathrm{H}_{2}\right.$ and 8'- $\left.\mathrm{H}_{2}\right), 4.85\left(\mathrm{~s}, \mathrm{NH}, \mathrm{NH}^{+}\right.$and $\left.\mathrm{H}_{2} \mathrm{O}\right), 5.58$ (broad d, $J=4.4 \mathrm{~Hz}$, 1H, 8-H), 7.52-7.60 (complex signal, 2H, 2-H and 7"-H), 7.77 (s, 1H, 4-H), superimposed in part 7.78 (d, J=5.6 Hz, 1H, 5"-H), 7.84 (m, 1H, 6"-H), 8.36-8.41 (complex signal, 2H, 1$\mathrm{H}$ and 8"-H); ${ }^{13} \mathrm{C}$ NMR (100.6 MHz, $\left.\mathrm{CD}_{3} \mathrm{OD}\right) \delta 21.9\left(\mathrm{CH}_{2}, \mathrm{C} 3 "\right), 23.0\left(\mathrm{CH}_{2}, \mathrm{C} 2 "\right), 23.5$ $\left(\mathrm{CH}_{3}, 9-\mathrm{CH}_{3}\right), 24.9\left(\mathrm{CH}_{2}, \mathrm{C} 1 "\right), 27.3(\mathrm{CH}, \mathrm{C} 11), 27.7\left(\mathrm{CH}_{2}\right)$ and $27.80\left(\mathrm{CH}_{2}\right)\left(\mathrm{C}^{\prime}\right.$ and $\left.\mathrm{C6} '\right)$, 27.84 (CH, C7), 29.3 (2 $\mathrm{CH}_{2}, \mathrm{C} 13$ and C4"), $30.2\left(\mathrm{CH}_{2}\right)$ and $30.3\left(\mathrm{CH}_{2}\right)\left(\mathrm{C} 4\right.$ 'and $\left.\mathrm{C}^{\prime}\right), 31.3$ $\left(\mathrm{CH}_{2}\right)$ and $31.5\left(\mathrm{CH}_{2}\right)\left(\mathrm{C}^{\prime}\right.$ and $\left.\mathrm{C}^{\prime}\right), 36.05\left(\mathrm{CH}_{2}\right)$ and $36.11\left(\mathrm{CH}_{2}\right)(\mathrm{C} 6$ and $\mathrm{C} 10), 49.5$ 
$\left(\mathrm{CH}_{2}\right)$ and $50.1\left(\mathrm{CH}_{2}\right)\left(\mathrm{C}^{\prime}\right.$ and $\left.\mathrm{C} 8 '\right), 112.8(\mathrm{C}), 115.6(\mathrm{C}), 117.0(\mathrm{C}), 117.6(\mathrm{C})(\mathrm{C} 11 \mathrm{a}$, C12a, C8"a, and C9"a), 119.1 (CH) and 120.1 (CH) (C4 and C5"), 125.2 (CH, C8), 126.3 (CH, C7"), 126.5 (CH, C8"), 126.7 (CH, C2), 129.5 (CH, C1), 134.1 (CH, C6"), 134.5 (C,

C9), 139.8 (C), 140.2 (C) and 141.0 (C) (C3, C4a and C10"a), 151.2 (C) and 151.7 (C) (C5a and C4"a), 156.9 (C) and 158.0 (C) (C12 and C9"). Anal. $\left(\mathrm{C}_{38} \mathrm{H}_{45} \mathrm{ClN}_{4} \cdot 2 \mathrm{HCl} \cdot 2 \mathrm{H}_{2} \mathrm{O}\right)$ C, $\mathrm{H}, \mathrm{N}, \mathrm{Cl}$.

3-Chloro-6,7,10,11-tetrahydro-9-methyl-12- $\{\{3-\{N-\{3-[(1,2,3,4-t e t r a h y d r o a c r i d i n-9-$ yl)amino]propyl $\}-N$-methylamino $\}$ propyl $\}$ amino $\}$-7,11-methanocycloocta[b]quinoline trihydrochloride [( \pm )-17d·3HCl]: $475 \mathrm{mg}, 57 \%$ yield (free base), light yellow oil: $R_{f} 0.46$

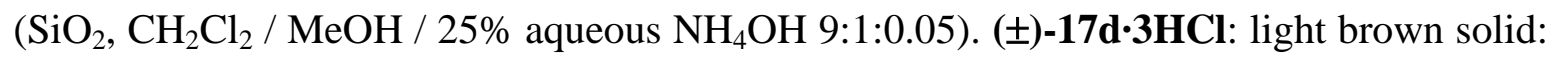
mp 271-272 ${ }^{\circ} \mathrm{C}(\mathrm{MeOH} / \mathrm{AcOEt} 1: 4) ; \mathrm{IR}(\mathrm{KBr}) v 3600-2500$ (max. at 3395, 3055, 3007, 2931, 2795 and 2701, N-H, N-H and C-H st), 1631, 1582 and 1520 (ar-C-C and ar-C-N st) $\mathrm{cm}^{-1}$; ${ }^{1} \mathrm{H}$ NMR (500 MHz, $\left.\mathrm{CD}_{3} \mathrm{OD}\right) \delta 1.58$ (s, 3H, 9- $\mathrm{CH}_{3}$ ), superimposed in part 1.93 (d, $J=18.0 \mathrm{~Hz}, 1 \mathrm{H}, 10-\mathrm{H}_{\text {endo }}$ ), 1.94-2.00 (complex signal, 5H, 13- $\mathrm{H}_{\text {syn }}, 2$ ''- $\mathrm{H}_{2}$ and 3"'-- $\mathrm{H}_{2}$ ), 2.08 (dm, $J=12.5 \mathrm{~Hz}, 1 \mathrm{H}, 13-\mathrm{H}_{\text {anti }}$ ), 2.33-2.45 (complex signal, 4H, 2'- $\mathrm{H}_{2}$ and 2"- $\mathrm{H}_{2}$ ), 2.59 (broad dd, $J=18.0 \mathrm{~Hz}, J^{\prime}=5.0 \mathrm{~Hz}, 1 \mathrm{H}, 10-\mathrm{H}_{\text {exo }}$ ), superimposed in part $2.79(\mathrm{~m}, 1 \mathrm{H}, 7-\mathrm{H})$,

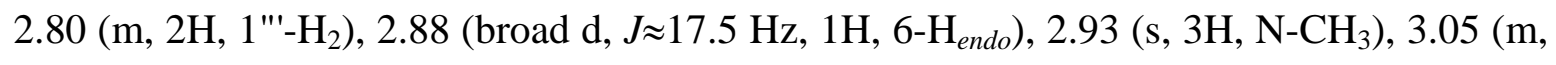
2H, 4"'- $\mathrm{H}_{2}$ ), 3.22 (dd, $J=17.5 \mathrm{~Hz}, J^{\prime}=5.5 \mathrm{~Hz}, 1 \mathrm{H}, 6-\mathrm{H}_{\text {exo }}$ ), 3.32-3.48 (complex signal, 4H,

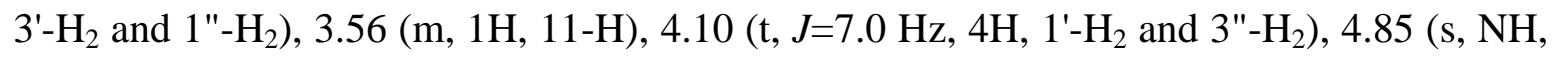
$\mathrm{NH}^{+}$and $\mathrm{H}_{2} \mathrm{O}$ ), 5.59 (broad d, $J=5.0 \mathrm{~Hz}, 1 \mathrm{H}, 8-\mathrm{H}$ ), 7.59 (dd, $J=9.5 \mathrm{~Hz}, J^{\prime}=2.0 \mathrm{~Hz}, 1 \mathrm{H}, 2-\mathrm{H}$ ), 
7.62 (ddm, $\left.J \approx J^{\prime} \approx 8.5 \mathrm{~Hz}, 1 \mathrm{H}, 7^{\prime \prime \prime}-\mathrm{H}\right)$, superimposed in part 7.77 (d, $\left.J \approx 2.0 \mathrm{~Hz}, 1 \mathrm{H}, 4-\mathrm{H}\right), 7.78$

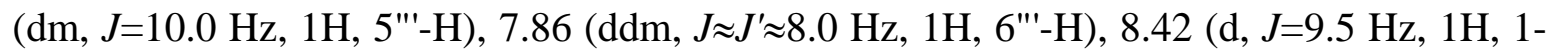
H), 8.44 (d, J=8.5 Hz, 1H, 8"'-H); ${ }^{13} \mathrm{C}$ NMR (75.4 MHz, CD $\left.{ }_{3} \mathrm{OD}\right) \delta 21.8\left(\mathrm{CH}_{2}, \mathrm{C} 3{ }^{\prime \prime}\right), 23.0$ $\left(\mathrm{CH}_{2}, \mathrm{C} 2 " '\right), 23.5\left(\mathrm{CH}_{3}, 9-\mathrm{CH}_{3}\right), 25.4\left(\mathrm{CH}_{2}, \mathrm{C} 1 " '\right), 26.4\left(\mathrm{CH}_{2}\right)$ and $26.6\left(\mathrm{CH}_{2}\right)(\mathrm{C} 2$ ' and C2"), 27.4 (CH, C11), 27.9 (CH, C7), $29.3\left(\mathrm{CH}_{2}\right)$ and $29.4\left(\mathrm{CH}_{2}\right)(\mathrm{C} 13$ and C4"'), 36.2 $\left(\mathrm{CH}_{2}, \mathrm{C} 10\right), 36.5\left(\mathrm{CH}_{2}, \mathrm{C} 6\right), 40.5\left(\mathrm{CH}_{3}, \mathrm{~N}-\mathrm{CH}_{3}\right), 45.9\left(\mathrm{CH}_{2}\right)$ and $46.5\left(\mathrm{CH}_{2}\right)\left(\mathrm{C} 1^{\prime}\right.$ and $\left.\mathrm{C} 3 "\right)$, $54.6\left(\mathrm{CH}_{2}\right)$ and $54.7\left(\mathrm{CH}_{2}\right)\left(\mathrm{C}^{\prime}\right.$ and $\left.\mathrm{C} 1 "\right), 113.5(\mathrm{C}), 115.7(\mathrm{C}), 117.2(\mathrm{C})$, and $118.2(\mathrm{C})$ (C11a, C12a, C8"'a, and C9"'a), 119.1 (CH) and 120.1 (CH) (C4 and C5"'), 125.0 (CH, C8), 126.2 (CH, C7"'), 126.7 (CH, C8"'), 127.1 (CH, C2), 129.3 (CH, C1), 134.0 (CH, C6"'), 134.6 (C, C9), 139.5 (C), 140.1 (C) and 140.7 (C) (C3, C4a and C10"'a), 151.7 (C) and 152.2 (C) (C5a and C4"'a), 156.7 (C) and 157.8 (C) (C12 and C9"'). Anal. $\left(\mathrm{C}_{37} \mathrm{H}_{44} \mathrm{ClN}_{5} \cdot 3 \mathrm{HCl} \cdot 2 \mathrm{H}_{2} \mathrm{O}\right) \mathrm{C}, \mathrm{H}, \mathrm{N}, \mathrm{Cl}$.

\section{3-Chloro-6,7,10,11-tetrahydro-9-methyl-12- $\{$ \{6-[(6-chloro-1,2,3,4-tetrahydroacridin-}

9-yl)amino]hexyl \}amino\}-7,11-methanocycloocta[b]quinoline dihydrochloride [( \pm )18a·2HCl]: $261 \mathrm{mg}, 34 \%$ yield (free base), yellowish oil: $R_{f} 0.59\left(\mathrm{SiO}_{2}, \mathrm{CH}_{2} \mathrm{Cl}_{2} / \mathrm{MeOH} /\right.$ 25\% aqueous $\mathrm{NH}_{4} \mathrm{OH}$ 9:1:0.05). (土)-18a·2HCl: light yellow solid: mp 239-240 ${ }^{\circ} \mathrm{C}(\mathrm{MeOH}$ / AcOEt 1:3); IR (KBr) v 3500-2500 (max. at 3397, 3230, 3048, 2927, 2860 and 2803, $\mathrm{N}^{+}$H, N-H and C-H st), 1631, 1582 and 1514 (ar-C-C and ar-C-N st) cm ${ }^{-1}$; ${ }^{1} \mathrm{H}$ NMR (400 $\mathrm{MHz}, \mathrm{CD}_{3} \mathrm{OD}$ ) $\delta 1.56$ (complex signal, 7H, 9- $\mathrm{CH}_{3}, 3$ '- $-\mathrm{H}_{2}$ and 4'- $\mathrm{H}_{2}$ ), 1.84-2.10 (complex

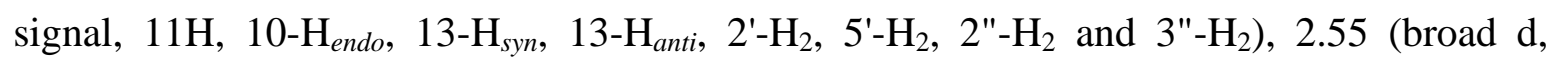


$\left.J=16.4 \mathrm{~Hz}, 1 \mathrm{H}, 10-\mathrm{H}_{\text {exo }}\right), 2.69$ (m, 2H, 1"- $\left.\mathrm{H}_{2}\right), 2.76$ (m, 1H, 7-H), 2.88 (d, J=17.6 Hz, 1H,

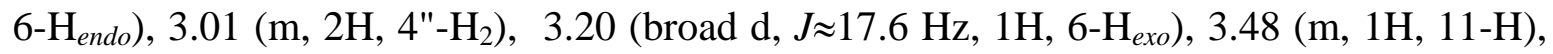
3.97 (complex signal, 4H, 1'- $\mathrm{H}_{2}$ and 6'- $\mathrm{H}_{2}$ ), 4.85 (s, $\mathrm{NH}, \mathrm{NH}^{+}$and $\mathrm{H}_{2} \mathrm{O}$ ), 5.58 (broad d, $J=4.4 \mathrm{~Hz}, 1 \mathrm{H}, 8-\mathrm{H}), 7.48-7.56$ (complex signal, 2H, 2-H and 7"-H), 7.77 (broad s, 1H) and 7.78 (broad s, 1H) (4-H and 5"-H), 8.35-8.43 (complex signal, 2H, 1-H and 8"-H); ${ }^{13} \mathrm{C}$ NMR (100.6 MHz, CD $3 \mathrm{OD}) \delta 21.8\left(\mathrm{CH}_{2}, \mathrm{C} 3 "\right), 22.9\left(\mathrm{CH}_{2}, \mathrm{C} 2 "\right), 23.5\left(\mathrm{CH}_{3}, 9-\mathrm{CH}_{3}\right), 24.9$

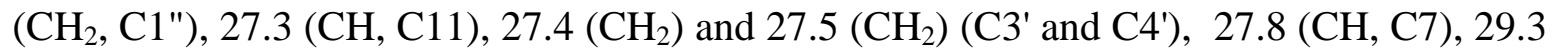
$\left(\mathrm{CH}_{2}\right)$ and $29.4\left(\mathrm{CH}_{2}\right)(\mathrm{C} 13$ and $\mathrm{C} 4 "), 31.2\left(\mathrm{CH}_{2}\right)$ and $31.3\left(\mathrm{CH}_{2}\right)\left(\mathrm{C}^{\prime}\right.$ and $\left.\mathrm{C}^{\prime}\right), 36.1\left(\mathrm{CH}_{2}\right.$, C10), $36.2\left(\mathrm{CH}_{2}, \mathrm{C} 6\right), 49.1\left(\mathrm{CH}_{2}\right)$ and $49.6\left(\mathrm{CH}_{2}\right)\left(\mathrm{C} 1{ }^{\prime}\right.$ and $\left.\mathrm{C}^{\prime}\right), 113.4(\mathrm{C}), 115.4(\mathrm{C}), 115.6$

(C) and 117.6 (C) (C11a, C12a, C8"a and C9"a), $119.12(\mathrm{CH})$ and $119.13(\mathrm{CH})$ (C4 and C5"), $125.2(\mathrm{CH}, \mathrm{C} 8), 126.7(\mathrm{CH})$ and $126.8(\mathrm{CH})(\mathrm{C} 2$ and $\mathrm{C7} "), 128.8(\mathrm{CH})$ and 129.5 (CH) (C1 and C8"), 134.5 (C, C9), 140.0 (C) and 140.1 (C) (C3 and C6"), 140.5 (C) and 140.9 (C) (C4a and C10"a), 151.2 (C) and 152.1 (C) (C5a and C4"a), 156.8 (C) and 157.7 (C) (C12 and C9"). Anal. $\left(\mathrm{C}_{36} \mathrm{H}_{40} \mathrm{Cl}_{2} \mathrm{~N}_{4} \cdot 2 \mathrm{HCl} \cdot 3 \mathrm{H}_{2} \mathrm{O}\right) \mathrm{C}, \mathrm{H}, \mathrm{N}, \mathrm{Cl}$.

\section{3-Chloro-6,7,10,11-tetrahydro-9-methyl-12- $\{$ \{-[(6-chloro-1,2,3,4-tetrahydroacridin-}

\section{9-yl)amino]heptyl\}amino $\}-7,11-m e t h a n o c y c l o o c t a[b] q u i n o l i n e ~ d i h y d r o c h l o r i d e ~[( \pm)-$}

18b·2HCl]: $317 \mathrm{mg}, 37 \%$ yield (free base), light yellow oil: $R_{f} 0.55\left(\mathrm{SiO}_{2}, \mathrm{CH}_{2} \mathrm{Cl}_{2} / \mathrm{MeOH}\right.$ / 25\% aqueous $\mathrm{NH}_{4} \mathrm{OH}$ 9:1:0.05). (土)-18b·2HCl: light yellow solid: mp 255-256 ${ }^{\circ} \mathrm{C}$ (MeOH / AcOEt 1:1); IR (KBr) v 3500-2500 (max. at 3410, 3256, 3048, 2929, 2857 and 2791, $\mathrm{N}^{+}-\mathrm{H}, \mathrm{N}-\mathrm{H}$ and C-H st), 1629, 1582 and 1520 (ar-C-C and ar-C-N st) cm ${ }^{-1}$; ${ }^{1} \mathrm{H}$ NMR 
(400 MHz, $\mathrm{CD}_{3} \mathrm{OD}$ ) $\delta 1.35-1.50$ (complex signal, 6H, 3'- $\mathrm{H}_{2}, 4^{\prime}-\mathrm{H}_{2}$ and 5'- $\mathrm{H}_{2}$ ), 1.57 (s, 3H, 9- $\mathrm{CH}_{3}$ ), 1.80-2.00 (complex signal, 10H, 10- $\mathrm{H}_{\text {endo, }}$ 13- $\mathrm{H}_{\text {syn }}, 2^{\prime}-\mathrm{H}_{2}, 6$ '- $\mathrm{H}_{2}, 2 "-\mathrm{H}_{2}$ and 3"- $\mathrm{H}_{2}$ ), 2.08 (broad d, $\left.J=11.2 \mathrm{~Hz}, 1 \mathrm{H}, 13-\mathrm{H}_{a n t i}\right), 2.55$ (broad d, J=16.8 Hz, 1H, 10- $\mathrm{H}_{\text {exo }}$ ), 2.68 (m,

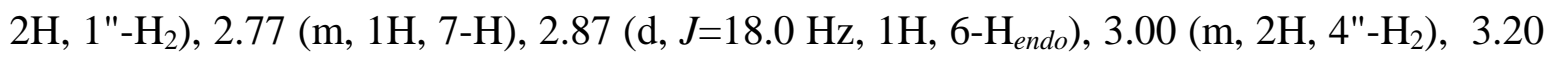
(dd, $\left.J=18.0 \mathrm{~Hz}, J^{\prime}=5.2 \mathrm{~Hz}, 1 \mathrm{H}, 6-\mathrm{H}_{\text {exo }}\right), 3.46(\mathrm{~m}, 1 \mathrm{H}, 11-\mathrm{H}), 3.95(\mathrm{t}, J=6.4 \mathrm{~Hz}, 2 \mathrm{H})$ and 3.98 (t, $J=7.2 \mathrm{~Hz}, 2 \mathrm{H})\left(1^{\prime}-\mathrm{H}_{2}\right.$ and $7^{\prime}-\mathrm{H}_{2}$ ), 4.85 (s, NH, $\mathrm{NH}^{+}$and $\mathrm{H}_{2} \mathrm{O}$ ), 5.58 (broad d, $J=4.4 \mathrm{~Hz}$, 1H, 8-H), 7.53 (d, J=8.8 Hz, 2H, 2-H and 7"-H), 7.77 (broad s, 1H) and 7.78 (broad s, 1H) (4-H and 5"-H), 8.38 (d, J=9.2 Hz, 2H, 1-H and 8"-H); ${ }^{13} \mathrm{C}$ NMR (100.6 MHz, CD $\left.3 \mathrm{OD}\right) \delta$ $21.8\left(\mathrm{CH}_{2}, \mathrm{C} 3 "\right), 22.9\left(\mathrm{CH}_{2}, \mathrm{C} 2 "\right), 23.5\left(\mathrm{CH}_{3}, 9-\mathrm{CH}_{3}\right), 24.8\left(\mathrm{CH}_{2}, \mathrm{C} 1 "\right), 27.3$ (CH, C11), 27.6 $\left(\mathrm{CH}_{2}\right)$ and $27.7\left(\mathrm{CH}_{2}\right)\left(\mathrm{C}^{\prime}\right.$ and $\left.\mathrm{C}^{\prime}\right), 27.8(\mathrm{CH}, \mathrm{C} 7), 29.3\left(2 \mathrm{CH}_{2}, \mathrm{C} 13\right.$ and $\left.\mathrm{C} 4 "\right), 29.9$ $\left(\mathrm{CH}_{2}, \mathrm{C} 4\right)$, $31.2\left(\mathrm{CH}_{2}\right)$ and $31.3\left(\mathrm{CH}_{2}\right)\left(\mathrm{C}^{\prime}\right.$ and $\left.\mathrm{C}^{\prime}\right), 36.05\left(\mathrm{CH}_{2}\right)$ and $36.13\left(\mathrm{CH}_{2}\right.$, $\mathrm{C} 6$ and C10), $48.6\left(\mathrm{CH}_{2}\right)$ and $49.3\left(\mathrm{CH}_{2}\right)\left(\mathrm{C}^{\prime}\right.$ and $\left.\mathrm{C} 7{ }^{\prime}\right), 113.3(\mathrm{C}), 115.4(\mathrm{C}), 115.6(\mathrm{C})$ and 117.6 (C) (C11a, C12a, C8"a and C9"a), 119.1 (2 CH, C4 and C5"), 125.2 (CH, C8), 126.7 (CH) and $126.8(\mathrm{CH})$ (C2 and C7"), $128.8(\mathrm{CH})$ and $129.5(\mathrm{CH})(\mathrm{C} 1$ and $\mathrm{C} 8 "), 134.5(\mathrm{C}, \mathrm{C} 9)$, 140.1 (C) and 140.2 (C) (C3 and C6"), 140.5 (C) and 141.0 (C) (C4a and C10"a), 151.2 (C) and 152.1 (C) (C5a and C4"a), 156.8 (C) and 157.8 (C) (C12 and C9"). Anal. $\left(\mathrm{C}_{37} \mathrm{H}_{42} \mathrm{Cl}_{2} \mathrm{~N}_{4} \cdot 2.1 \mathrm{HCl} \cdot 3 \mathrm{H}_{2} \mathrm{O}\right) \mathrm{C}, \mathrm{H}, \mathrm{N}, \mathrm{Cl}$.

3-Chloro-6,7,10,11-tetrahydro-9-methyl-12- $\{$ \{8-[(6-chloro-1,2,3,4-tetrahydroacridin9-yl)amino] octyl \}amino $\}$-7,11-methanocycloocta[b]quinoline dihydrochloride [( \pm )18c·2HCl]: $317 \mathrm{mg}, 52 \%$ yield (free base), light yellow oil: $R_{f} 0.46\left(\mathrm{SiO}_{2}, \mathrm{CH}_{2} \mathrm{Cl}_{2} / \mathrm{MeOH}\right.$ 
/ 25\% aqueous $\mathrm{NH}_{4} \mathrm{OH}$ 9:1:0.05). ( \pm )-18c·2HCl: light brown solid: mp 228-229 ${ }^{\circ} \mathrm{C}(\mathrm{MeOH}$ / AcOEt 1:3); IR (KBr) v 3600-2500 (max. at 3415, 3244, 3120, 3046, 2928, 2853 and 2793, $\mathrm{N}^{+}-\mathrm{H}, \mathrm{N}-\mathrm{H}$ and C-H st), 1629, 1584 and 1522 (ar-C-C and ar-C-N st) cm ${ }^{-1}$; ${ }^{1} \mathrm{H}$ NMR (400 MHz, $\mathrm{CD}_{3} \mathrm{OD}$ ) $\delta 1.37-1.49$ (complex signal, 8H, 3'- $\mathrm{H}_{2}, 4^{\prime}-\mathrm{H}_{2}, 5^{\prime}-\mathrm{H}_{2}$ and 6'- $\mathrm{H}_{2}$ ), 1.57 (s, 3H, 9-- $\mathrm{CH}_{3}$ ), 1.79-2.00 (complex signal, 10H, 10- $\mathrm{H}_{\text {endo, }}$, 13- $\mathrm{H}_{\text {syn }}, 2^{\prime}-\mathrm{H}_{2}, 7^{\prime}-\mathrm{H}_{2}, 2 "-\mathrm{H}_{2}$ and 3"- $\mathrm{H}_{2}$ ), 2.08 (dm, $\left.J=12.8 \mathrm{~Hz}, 1 \mathrm{H}, 13-\mathrm{H}_{a n t i}\right), 2.54$ (broad dd, $J=17.4 \mathrm{~Hz}, J^{\prime}=4.6 \mathrm{~Hz}, 1 \mathrm{H}, 10-$ $\mathrm{H}_{\text {exo }}$ ), 2.67 (m, 2H, 1"-- $\mathrm{H}_{2}$ ), 2.76 (m, 1H, 7-H), 2.87 (broad d, $J=18.0 \mathrm{~Hz}, 1 \mathrm{H}, 6-\mathrm{H}_{\text {endo }}$ ), 3.00 (m, 2H, 4"-- $\mathrm{H}_{2}$ ), 3.20 (dd, J=18.0 Hz, J'=5.6 Hz, 1H, 6- $\mathrm{H}_{\text {exo }}$ ), 3.45 (m, 1H, 11-H), 3.94 (t, $J=7.6 \mathrm{~Hz}, 2 \mathrm{H})$ and $3.97(\mathrm{t}, J=7.2 \mathrm{~Hz}, 2 \mathrm{H})\left(1^{\prime}-\mathrm{H}_{2}\right.$ and $\left.8^{\prime}-\mathrm{H}_{2}\right), 4.85\left(\mathrm{~s}, \mathrm{NH}, \mathrm{NH}^{+}\right.$and $\left.\mathrm{H}_{2} \mathrm{O}\right)$, 5.59 (broad d, $J=4.8 \mathrm{~Hz}, 1 \mathrm{H}, 8-\mathrm{H}$ ), 7.54 (dd, $J=9.2 \mathrm{~Hz}, J^{\prime} \approx 2.4 \mathrm{~Hz}, 1 \mathrm{H}$ ) and 7.55 (dd, $J=9.2$ $\left.\mathrm{Hz}, J^{\prime}=2.4 \mathrm{~Hz}, 1 \mathrm{H}\right)(2-\mathrm{H}$ and 7"-H), $7.77(\mathrm{~d}, J=2.4 \mathrm{~Hz}, 1 \mathrm{H})$ and $7.78(\mathrm{~d}, J=2.4 \mathrm{~Hz}, 1 \mathrm{H})(4-\mathrm{H}$ and 5"-H), 8.38 (d, $J=9.2 \mathrm{~Hz}, 1 \mathrm{H})$ and 8.39 (d, $J=9.2 \mathrm{~Hz}, 1 \mathrm{H})(1-\mathrm{H}$ and $8 "-\mathrm{H}) ;{ }^{13} \mathrm{C}$ NMR (100.6 MHz, $\left.\mathrm{CD}_{3} \mathrm{OD}\right) \delta 21.8\left(\mathrm{CH}_{2}, \mathrm{C} 3 "\right), 22.9\left(\mathrm{CH}_{2}, \mathrm{C} 2 "\right), 23.5\left(\mathrm{CH}_{3}, 9-\mathrm{CH}_{3}\right), 24.7\left(\mathrm{CH}_{2}\right.$, C1"), $27.3(\mathrm{CH}, \mathrm{C} 11), 27.7\left(\mathrm{CH}_{2}\right)$ and $27.78\left(\mathrm{CH}_{2}\right)(\mathrm{C} 3$ ' and $\mathrm{C6}$ '), $27.84(\mathrm{CH}, \mathrm{C} 7), 29.29$ $\left(\mathrm{CH}_{2}\right)$ and $29.31\left(\mathrm{CH}_{2}\right)(\mathrm{C} 13$ and $\mathrm{C} 4 "), 30.18\left(\mathrm{CH}_{2}\right)$ and $30.24\left(\mathrm{CH}_{2}\right)\left(\mathrm{C}^{\prime}\right.$ and $\left.\mathrm{C}^{\prime}\right), 31.3$ $\left(\mathrm{CH}_{2}\right)$ and $31.4\left(\mathrm{CH}_{2}\right)\left(\mathrm{C}^{\prime}\right.$ and $\left.\mathrm{C}^{\prime}\right), 36.0\left(\mathrm{CH}_{2}, \mathrm{C} 10\right), 36.1\left(\mathrm{CH}_{2}, \mathrm{C} 6\right), 48.8\left(\mathrm{CH}_{2}\right)$ and 49.2 (CH2) (C1' and C8'), 113.3 (C), 115.4 (C), 115.6 (C) and 117.6 (C) (C11a, C12a, C8"a and C9"a), 119.2 (2 CH, C4 and C5"), 125.2 (CH, C8), $126.6(\mathrm{CH})$ and $126.8(\mathrm{CH})(\mathrm{C} 2$ and C7"), $128.8(\mathrm{CH})$ and $129.5(\mathrm{CH})$ (C1 and C8"), 134.5 (C, C9), 140.1 (C) and 140.2 (C) (C3 and C6"), 140.5 (C) and 141.0 (C) (C4a and C10"a), 151.2 (C) and 152.1 (C) (C5a and 
C4"a), 156.9 (C) and 157.8 (C) (C12 and C9"). Anal. $\left(\mathrm{C}_{38} \mathrm{H}_{44} \mathrm{Cl}_{2} \mathrm{~N}_{4} \cdot 2 \mathrm{HCl} \cdot 3.2 \mathrm{H}_{2} \mathrm{O}\right) \mathrm{C}, \mathrm{H}, \mathrm{N}$, Cl.

3-Chloro-6,7,10,11-tetrahydro-9-methyl-12- $\{\{3-\{N-\{3-[(6-c h l o r o-1,2,3,4-$ tetrahydroacridin-9-yl)amino]propyl $\}-N$-methylamino $\}$ propyl $\}$ amino $\}-7,11-$

methanocycloocta[b]quinoline trihydrochloride [( \pm )-18d·3HCl]: $252 \mathrm{mg}, 33 \%$ yield (free base), light yellow oil: $R_{f} 0.57\left(\mathrm{SiO}_{2}, \mathrm{CH}_{2} \mathrm{Cl}_{2} / \mathrm{MeOH} / 25 \%\right.$ aqueous $\mathrm{NH}_{4} \mathrm{OH}$ 9:1:0.05). ( \pm )-18d·3HCl: light brown solid: mp 220-221 ${ }^{\circ} \mathrm{C}(\mathrm{MeOH} /$ AcOEt 1:4); IR (KBr) $v$ 3600-2500 (max. at 3420, 3262, 3054, 2999, 2930 and 2790, $\mathrm{N}^{+}-\mathrm{H}, \mathrm{N}-\mathrm{H}$ and C-H st), 1629, 1581 and 1512 (ar-C-C and ar-C-N st) cm ${ }^{-1} ;{ }^{1} \mathrm{H}$ NMR (500 MHz, CD ${ }_{3} \mathrm{OD}$ ) $\delta 1.58$ (s, 3H, 9- $\left.\mathrm{CH}_{3}\right)$, superimposed in part $1.93\left(\mathrm{~d}, J=18.0 \mathrm{~Hz}, 1 \mathrm{H}, 10-\mathrm{H}_{\text {endo }}\right.$ ), $1.94-2.00$

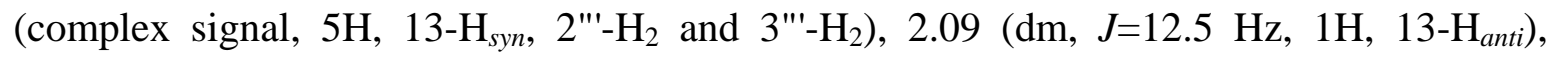
2.32-2.46 (complex signal, 4H, 2'- $\mathrm{H}_{2}$ and 2"- $\mathrm{H}_{2}$ ), $2.58\left(\mathrm{dm}, J \approx 18.0 \mathrm{~Hz}, 1 \mathrm{H}, 10-\mathrm{H}_{\text {exo }}\right.$ ), 2.76

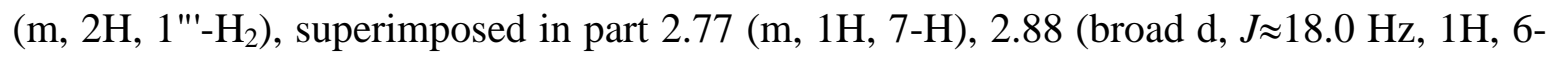

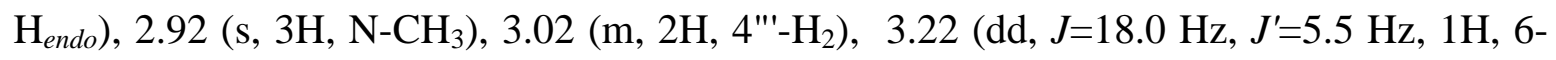
$\mathrm{H}_{\text {exo }}$ ), 3.26-3.46 (complex signal, 4H, 3'- $\mathrm{H}_{2}$ and 1"- $\mathrm{H}_{2}$ ), 3.55 (m, 1H, 11-H), 4.09 (complex signal, 4H, 1'- $\mathrm{H}_{2}$ and 3"-- $\mathrm{H}_{2}$ ), 4.87 (s, $\mathrm{NH}, \mathrm{NH}^{+}$and $\mathrm{H}_{2} \mathrm{O}$ ), 5.59 (broad d, J=4.5 Hz, 1H, 8H), 7.58 (broad dd, $J \approx 9.0 \mathrm{~Hz}, J^{\prime}=2.0 \mathrm{~Hz}, 2 \mathrm{H}, 2-\mathrm{H}$ and 7"'-H), 7.76 (d, J=2.0 Hz, 1H) and 7.77 (d, $J=2.0 \mathrm{~Hz}, 1 \mathrm{H})(4-\mathrm{H}$ and 5"'-H), 8.41 (d, $J=9.5 \mathrm{~Hz}, 1 \mathrm{H})$ and 8.44 (d, J=9.0 Hz, 1H) (1-H and 8"'-H); ${ }^{13} \mathrm{C}$ NMR (75.4 MHz, $\left.\mathrm{CD}_{3} \mathrm{OD}\right) \delta 21.7\left(\mathrm{CH}_{2}, \mathrm{C} 3{ }^{\prime \prime}\right), 22.9\left(\mathrm{CH}_{2}, \mathrm{C} 2 " '\right), 23.5$ ( $\left.\mathrm{CH}_{3}, 9-\mathrm{CH}_{3}\right), 25.3\left(\mathrm{CH}_{2}, \mathrm{C} 1 " '\right), 26.4$ (2 $\mathrm{CH}_{2}, \mathrm{C} 2$ ' and C2"), $27.4(\mathrm{CH}, \mathrm{C} 11), 27.9(\mathrm{CH}, \mathrm{C} 7)$, 
$29.3\left(\mathrm{CH}_{2}\right)$ and $29.5\left(\mathrm{CH}_{2}\right)$ (C13 and C4"'), $36.1\left(\mathrm{CH}_{2}, \mathrm{C} 10\right), 36.4\left(\mathrm{CH}_{2}, \mathrm{C} 6\right), 40.5\left(\mathrm{CH}_{3}, \mathrm{~N}-\right.$ $\left.\mathrm{CH}_{3}\right), 46.0\left(\mathrm{CH}_{2}\right)$ and $46.6\left(\mathrm{CH}_{2}\right)\left(\mathrm{C}^{\prime}\right.$ and $\left.\mathrm{C} 3 "\right), 54.7\left(2 \mathrm{CH}_{2}, \mathrm{C} 3\right.$ ' and $\left.\mathrm{C} 1 "\right), 113.9(\mathrm{C})$, 115.6 (C), 115.7 (C) and 118.2 (C) (C11a, C12a, C8"'a and C9"'a), 119.1 (2 CH, C4 and C5"'), $125.0(\mathrm{CH}, \mathrm{C} 8), 127.08(\mathrm{CH})$ and $127.13(\mathrm{CH})(\mathrm{C} 2$ and $\mathrm{C} 7 " '), 128.6(\mathrm{CH})$ and 129.3 (CH) (C1 and C8"'), 134.6 (C, C9), 140.0 (C) and 140.1 (C) (C3 and C6"'), 140.2 (C) and 140.8 (C) (C4a and C10"'a), 151.7 (C) and 152.7 (C) (C5a and C4"'a), 156.7 (C) and 157.7 (C) (C12 and C9"'). Anal. $\left(\mathrm{C}_{37} \mathrm{H}_{43} \mathrm{Cl}_{2} \mathrm{~N}_{5} \cdot 3 \mathrm{HCl} \cdot 4 \mathrm{H}_{2} \mathrm{O}\right) \mathrm{C}, \mathrm{H}, \mathrm{N}, \mathrm{Cl}$.

Biochemical studies. AChE inhibitory activity was evaluated spectrophotometrically at 25 ${ }^{\circ} \mathrm{C}$ by the method of Ellman, ${ }^{13}$ using AChE from bovine or human erythrocytes and acetylthiocholine iodide ( $0.53 \mathrm{mM}$ or $0.27 \mathrm{mM}$ for bovine and human AChE, respectively) as substrate. The reaction took place in a final volume of $3 \mathrm{~mL}$ of $0.1 \mathrm{M}$ phosphate-buffered solution $\mathrm{pH}$ 8.0, containing 0.025 units of $\mathrm{AChE}$ and $333 \mu \mathrm{M}$ 5,5'-dithiobis(2-nitrobenzoic) acid (DTNB) solution used to produce the yellow anion of 5-thio-2-nitrobenzoic acid. Inhibition curves were performed in triplicate by incubating with at least 12 concentrations of inhibitor for $15 \mathrm{~min}$. One triplicate sample without inhibitor was always present to yield $100 \%$ of AChE activity. The reaction was stopped by the addition of $100 \mu \mathrm{L} 1 \mathrm{mM}$ eserine, and the color production was measured at $414 \mathrm{~nm}$. In some experiments the timedependence of the inhibitory processes was determined for the compounds with at least 12 increasing concentrations of inhibitor after an incubation period of $45 \mathrm{~min}$. BChE inhibitory activity determinations were carried out similarly, using 0.035 unit of human serum BChE 
and $0.56 \mathrm{mM}$ butyrylthiocholine, instead of AChE and acetylthiocholine, in a final volume of $1 \mathrm{~mL}$.

Data from concentration-inhibition experiments of the inhibitors were calculated by nonlinear regression analysis, using the GraphPad Prism program package (GraphPad Software; San Diego, USA), which gave estimates of the IC $_{50}$ (concentration of drug producing $50 \%$ of enzyme activity inhibition). Results are expressed as mean \pm S.E.M. of at least 4 experiments performed in triplicate. DTNB, acetylthiocholine, butyrylthiocholine, and the enzymes were purchased from Sigma and eserine from Fluka. 


\section{Additional References}

19. Aguado, F.; Badía, A.; Baños, J. E.; Bosch, F.; Bozzo, C.; Camps, P.; Contreras, J.; Dierssen, M.; Escolano, C.; Görbig, D. M.; Muñoz-Torrero, D.; Pujol, M. D.; Simón, M.; Vázquez, M. T.; Vivas, N. M. Synthesis and Evaluation of TacrineRelated Compounds for the Treatment of Alzheimer’s Disease. Eur. J. Med. Chem. 1994, 29, 205-221.

20. Savini, L.; Campiani, G.; Gaeta, A.; Pellerano, C.; Fattorusso, C.; Chiasserini, L.; Fedorko, J. M.; Saxena, A. Novel and Potent Tacrine-Related Hetero- and Homobivalent Ligands for Acetylcholinesterase and Butyrylcholinesterase. Bioorg. Med. Chem. Lett. 2001, 11, 1779-1782.

21. Apelt, J.; Ligneau, X.; Pertz, H. H.; Arrang, J. -M.; Ganellin, C. R.; Schwartz, J. C.; Schunack, W.; Stark, H. Development of a New Class of Nonimidazole Histamine $\mathrm{H}_{3}$ Receptor Ligands with Combined Inhibitory Histamine $\mathrm{N}$ Methyltransferase Activity. J. Med. Chem. 2002, 45, 1128-1141. 


\section{Appendix}

\begin{tabular}{|c|c|c|c|c|c|c|c|c|c|}
\hline \multirow[t]{2}{*}{ Compd } & \multirow{2}{*}{$\begin{array}{l}\text { Molecular } \\
\text { Formula }\end{array}$} & \multicolumn{4}{|c|}{ Calculated } & \multicolumn{4}{|c|}{ Found } \\
\hline & & $\mathrm{C}$ & $\mathrm{H}$ & $\mathrm{N}$ & $\mathrm{Cl}$ & $\mathrm{C}$ & $\mathrm{H}$ & $\mathrm{N}$ & $\mathrm{Cl}$ \\
\hline$( \pm)-6$ & $\mathrm{C}_{18} \mathrm{H}_{19} \mathrm{NO} \cdot 0.4 \mathrm{H}_{2} \mathrm{O}$ & 79.32 & 7.32 & 5.14 & & 79.23 & 7.32 & 5.30 & \\
\hline$( \pm)-8$ & $\mathrm{C}_{18} \mathrm{H}_{18} \mathrm{ClN}$ & 76.18 & 6.39 & 4.94 & 12.49 & 76.29 & 6.39 & 4.94 & 12.55 \\
\hline$( \pm)-12 \cdot 2 \mathrm{HCl} \cdot 2 \mathrm{H}_{2} \mathrm{O} \cdot 0.5 \mathrm{AcOEt}$ & $\mathrm{C}_{39} \mathrm{H}_{48} \mathrm{~N}_{4} \cdot 2 \mathrm{HCl} \cdot 2 \mathrm{H}_{2} \mathrm{O} \cdot 0.5 \mathrm{AcOEt}$ & 67.85 & 8.05 & 7.72 & & 67.88 & 8.02 & 7.33 & \\
\hline 15d $\cdot 2 \mathrm{HCl} \cdot \mathrm{H}_{2} \mathrm{O} \cdot 0.2 \mathrm{AcOEt}$ & $\mathrm{C}_{20} \mathrm{H}_{28} \mathrm{ClN}_{3} \cdot 2 \mathrm{HCl} \cdot \mathrm{H}_{2} \mathrm{O} \cdot 0.2 \mathrm{AcOEt}$ & 54.97 & 7.45 & 9.25 & 23.40 & 54.95 & 7.52 & 9.30 & 23.22 \\
\hline$( \pm)-17 \mathbf{a} \cdot 2.1 \mathrm{HCl} \cdot 3.5 \mathrm{H}_{2} \mathrm{O}$ & $\mathrm{C}_{36} \mathrm{H}_{41} \mathrm{ClN}_{4} \cdot 2.1 \mathrm{HCl} \cdot 3.5 \mathrm{H}_{2} \mathrm{O}$ & 61.35 & 7.16 & 7.95 & 15.59 & 61.47 & 7.16 & 7.68 & 15.68 \\
\hline$( \pm)-17 \mathbf{b} \cdot 2 \mathrm{HCl} \cdot 3.2 \mathrm{H}_{2} \mathrm{O} \cdot 0.1 \mathrm{AcOEt}$ & $\mathrm{C}_{37} \mathrm{H}_{43} \mathrm{ClN}_{4} \cdot 2 \mathrm{HCl} \cdot 3.2 \mathrm{H}_{2} \mathrm{O} \cdot 0.1 \mathrm{AcOEt}$ & 62.51 & 7.32 & 7.80 & 14.80 & 62.57 & 7.03 & 7.40 & 15.08 \\
\hline$( \pm)-\mathbf{1 7} \cdot 2 \mathrm{HCl} \cdot 2 \mathrm{H}_{2} \mathrm{O}$ & $\mathrm{C}_{38} \mathrm{H}_{45} \mathrm{ClN}_{4} \cdot 2 \mathrm{HCl} \cdot 2 \mathrm{H}_{2} \mathrm{O}$ & 65.00 & 7.32 & 7.98 & 15.15 & 64.68 & 7.27 & 7.72 & 15.08 \\
\hline$( \pm)-\mathbf{1 7 d} \cdot 3 \mathrm{HCl} \cdot 2 \mathrm{H}_{2} \mathrm{O}$ & $\mathrm{C}_{37} \mathrm{H}_{44} \mathrm{ClN}_{5} \cdot 3 \mathrm{HCl} \cdot 2 \mathrm{H}_{2} \mathrm{O}$ & 60.08 & 6.95 & 9.47 & 19.17 & 60.02 & 6.97 & 9.33 & 19.51 \\
\hline$( \pm)-\mathbf{1 8 a} \cdot 2 \mathrm{HCl} \cdot 3 \mathrm{H}_{2} \mathrm{O}$ & $\mathrm{C}_{36} \mathrm{H}_{40} \mathrm{Cl}_{2} \mathrm{~N}_{4} \cdot 2 \mathrm{HCl} \cdot 3 \mathrm{H}_{2} \mathrm{O}$ & 59.51 & 6.66 & 7.71 & 19.52 & 59.62 & 6.53 & 7.31 & 19.13 \\
\hline$( \pm)-\mathbf{1 8 b} \cdot 2.1 \mathrm{HCl} \cdot 3 \mathrm{H}_{2} \mathrm{O}$ & $\mathrm{C}_{37} \mathrm{H}_{42} \mathrm{Cl}_{2} \mathrm{~N}_{4} \cdot 2.1 \mathrm{HCl} \cdot 3 \mathrm{H}_{2} \mathrm{O}$ & 59.71 & 6.79 & 7.53 & 19.53 & 59.54 & 6.54 & 7.27 & 19.78 \\
\hline$( \pm)-18 \mathrm{c} \cdot 2 \mathrm{HCl} \cdot 3.2 \mathrm{H}_{2} \mathrm{O}$ & $\mathrm{C}_{38} \mathrm{H}_{44} \mathrm{Cl}_{2} \mathrm{~N}_{4} \cdot 2 \mathrm{HCl} \cdot 3.2 \mathrm{H}_{2} \mathrm{O}$ & 60.19 & 6.97 & 7.39 & 18.70 & 59.95 & 6.61 & 7.28 & 18.96 \\
\hline$( \pm)-\mathbf{1 8 d} \cdot 3 \mathrm{HCl} \cdot 4 \mathrm{H}_{2} \mathrm{O}$ & $\mathrm{C}_{37} \mathrm{H}_{43} \mathrm{Cl}_{2} \mathrm{~N}_{5} \cdot 3 \mathrm{HCl} \cdot 4 \mathrm{H}_{2} \mathrm{O}$ & 54.86 & 6.72 & 8.64 & 21.88 & 55.10 & 6.61 & 8.76 & 21.69 \\
\hline
\end{tabular}

